\title{
Properties of alkali-halide salt solutions about polarizable nanoparticle solutes for different ion models
}

\author{
Aaron Wynveen* \\ Institut für Theoretische Physik II, Heinrich-Heine-Universität \\ Universitätsstraße 1, D-40225 Düsseldorf, Germany and \\ School of Physics and Astronomy, University of Minnesota \\ Minneapolis, Minnesota 55455, USA \\ Fernando Bresme ${ }^{\dagger}$ \\ Department of Chemistry, Imperial College London, \\ Exhibition Road, London SW7 2AZ, United Kingdom
}

(Dated: September 18, 2010)

\begin{abstract}
We investigate the distributions of various salts about large hydrophobic polarizable solutes in aqueous electrolyte solutions. The solutes are modeled as nanometer-sized cylindrical objects, a scale relevant to bio-molecules and nano-materials, and particularly high aspect ratio nanoparticles. Interactions, including image charge forces arising from the finite polarizability of the solute, between explicit solvent/ions and the solute are computed explicitly using a molecular dynamics simulation methodology we have recently introduced. Comparisons are made between several salt species and different models of the force fields for each ionic component of the salt. We find evidence that both small cations, $\mathrm{Li}^{+}$, and large anions, $\mathrm{I}^{-}$, adsorb at hydrophobic interfaces. Our results indicate that the ion structure about the solute is strongly dependent on the force field investigated, suggesting that ion selectivity is quite sensitive to the respective parameters defining the ion's size and binding energy as well as to the polarizability of the solute.
\end{abstract}

\section{INTRODUCTION}

The behavior of ions at interfaces is relevant to a wide range of problems, from stability of foams and nucleation, to the regulation of interactions between materials and biomolecules.[1-4] A traditional approach to deal with ionic mediated interactions has been the Poisson-Boltzmann (PB) theory, which forms the basis of the DLVO theory. [5, 6] In the PB theory, water is included as a dielectric continuum, and the ions are modeled as point charges. This theory has been useful for understanding the stability of thin films, $[1]$ and it has provided important clues on the interaction between DNA molecules.[2] It is fair to say that within its simplicity, this has been the most successful theory to explain colloidal suspension stability. However, there have been a number of studies that clearly indicate that the approximations introduced in this mean field theory become inaccurate near surfaces, and in general at interfaces, where the properties of water deviate from those of a continuum dielectric. As a matter of fact recent computer simulation investigations have illustrated how confinement can modify the dielectric properties of water.[7] It has been suggested that such modification can introduce a contribution to the so-called hydration force, which appears in conditions where water is confined in narrow spaces, typically of the order of a few nanometers. [7-9] This force has been observed in many systems,[10] and its physical origin has been the subject of a hot debate.[11, 12] Starting from the seminal work of Marcelja and Radic,[13] who explained hydration forces in terms of water polarization, several other theoretical approaches have been developed.[14, 15] One important outcome of these studies is that the molecular properties of water cannot be ignored for distances from the surface of the order of $1 \mathrm{~nm}$. Similarly, in the context of ionic solutions, ion size as well as dispersion interactions become a relevant variable, especially for concentrations above the physiological one $(\sim 0.15 \mathrm{M})$, where the ionic interactions are strongly screened. The relevance of dispersion interactions in ion selectivity has been discussed by Ninham and coworkers, who have proposed corrections to the traditional DLVO theory to account for such effects.[16, 17]

Ion selectivity phenomena have been known for many years. They were firstly exposed by Hofmeister in a celebrated article.[18] A recent illustration of ion selectivity near interfaces has been shown by Jungwirth and Tobias (JT), who investigated several alkali halides near a water surface.[3] Traditionally it has been considered that ions are depleted from the aqueous surface. This idea was first put on a firm theoretical basis by Onsager and Samaras (OS),[19] using

\footnotetext{
*Electronic address: a.wynveen@googlemail.com

${ }^{\dagger}$ Electronic address: f.bresme@imperial.ac.uk
} 
a mean field approach that neglects the molecular character of water as well as the size and dispersion interactions of the ions. This theory remains qualitatively correct for some alkali halides, typically those involving small weakly polarizable anions. However, as described in this article, this theory fails to capture, e.g., the physical behavior of very small cations, $\mathrm{Li}^{+}$. Corrections for polarizable ions have been proposed very recently. [20] As shown by JT, large ions can exhibit adsorption at the water surface. The microscopic details on the origin of this surface adsorption are still under discussion. There is evidence that ion polarizability[3] can induce adsorption, but there is also clear evidence of a correlation between ion size and charge and ion segregation.[21-26]

The main conclusion from the discussion above is that the investigation of an aqueous solution near surfaces requires the inclusion of both explicit water as well as the inclusion of the ion specificity through ion size and ion-surface interactions. These effects cannot be accounted for by theories that depend on ion valency only.

In this paper we discuss structural studies of aqueous solutions next to neutral polarizable nanoparticles. This situation is of interest in materials science and biophysics, e.g, nanotubes and biomolecules. One main feature of these systems is the existence of a dielectric discontinuity between the solute and the solvent. In the context of theoretical and simulation investigations, such discontinuities can be handled through an image charge approach. This idea was employed by OS to rationalize the increase in the surface tension with increasing salt concentrations in aqueous solutions.[19] In the context of this mean field approach, which ignores the molecular character of water, the strength of the induced polarization on the substrate and the character of the resulting ion-substrate interactions, being either repulsive or attractive, is determined by a difference in the permittivities of the two media. Such an approach has been considered in combination with more refined models of the ionic dispersion interactions in theoretical and simulation studies of aqueous solutions.[16, 17, 27-31] In general, these approaches neglect the molecular structure of water and, consequently, the reported deviations of the dielectric properties of interfacial water with respect to bulk water. $[7,32]$

Here, we are interested in modeling the dielectric discontinuities arising in aqueous solutions at the solute-solvent interface, including both water's molecular structure and ion specificity effects. Such an investigation can be performed using a model we have introduced recently to investigate the influence of solute polarization on hydrophobic forces. [3335] Extending this model to handle aqueous solutions requires the incorporation of ion-ion and ion-solute interactions. It has been noticed in theoretical work that ion selectivity is fairly sensitive to the ion dispersion interactions. Similarly, recent simulation work has shown that the properties of highly concentrated ionic solutions are sensitive to the force fields employed to model the ion interactions. [36] Although such differences are expected to be much smaller at lower concentrations, e.g., below physiological ones, the sensitivity of ion selectivity to the dispersion interaction makes it necessary to analyze the dependence of this property with the ion force field. In this paper, then, we address two main questions: 1) how does the ion distribution near a solute depend on solute polarizability? and 2) how does the ion distribution change for different ion species?

The paper is structured as follows. First we discuss the implementation of our dielectric model to simulate aqueous solutions along with details of the different ion force fields used in this work. A discussion of the results for various aqueous salt solutions next to dielectric solutes as a function of solute polarizability follows. The main conclusions close the paper.

\section{MODEL AND SIMULATION DETAILS}

We have carried out molecular dynamics simulations of polarizable cylindrical solutes in ionic solutions, considering both water, which we model using the simple point charge/extended (SPC/E) potential, and ions explicitly. This solute geometry is relevant in a number of problems of interest in biophysics and materials science including DNA, nanotubes, nanorods and protein bundles. The polarizable solute is modeled as a linear dielectric medium with permittivity $\varepsilon_{i n}$. Following our previous work, induced surface charges on the solute are modeled using a fixed grid of charges, which are located near the cylinder surface and whose magnitudes vary with the charge distribution of the external charges (water and ions).[33, 35, 37] At each time step, the induced charge on the cylinder due to any external charge, i.e., water partial charges, ion charges, or induced charge on another cylinder, is determined at every point on the cylinder surface using a look-up table. The look-up table was constructed by computing the induced surface charge on a cylinder, due to an external point charge, as a function of the solute permittivity, $\varepsilon_{\text {in }}$. [33] This induced surface charge is represented by an evenly-spaced grid, with a $1 \times 1 \AA^{2}$ mesh in the $(\phi, z)$ plane, which envelops the cylinder. Every time step, the grid charges are altered in response to the new positions of the external charges. This method replicates the dielectric discontinuity existing between the solute and the solution. In the present study, the surface charge grid was situated at a solute radius $R_{c}=7 \AA$, a size relevant to many biomolecules and nanorods. We note that the parameter $R_{c}$ in combination with the short range repulsive interaction defined below defines the effective diameter of the solute.

The calculation of the induced charge due to the external charges has been refined in the present study as compared 
to previous work.[33-35] Here, we have optimized the spatial interpolation algorithm for calculating the induced charges to provide a better representation of the dielectric discontinuity at the solute surface. Furthermore, to account for the different ion sizes and to prevent the collapse of external charges on the induced grid charges, we employed a repulsive potential of the form:

$$
V_{c y l, \alpha}(r)=\frac{3 \sqrt{3}}{2} \epsilon_{c y l, \alpha}\left[\left(\frac{\sigma_{c y l, \alpha}}{r-R_{c}}\right)^{9}-\left(\frac{\sigma_{c y l, \alpha}}{r-R_{c}}\right)^{3}+\frac{2}{3 \sqrt{3}}\right],
$$

with the potential being cutoff at $r=R_{c}+\sqrt[6]{3} \sigma_{c y l, \alpha}$ so that it is fully repulsive. We do not include an attractive mean-field term that accounts for the non-zero frequency dispersion interaction so as to isolate the effects of the zero-frequency image charge contribution. The normal, Lorentz-Berthelot, combining rules, $\epsilon_{c y l, \alpha}=\sqrt{\epsilon_{c y l} \epsilon_{\alpha}}$ and $\sigma_{c y l, \alpha}=\sigma_{\alpha} / 2$ (such that the distance of closest approach of the ion to the dielectric discontinuity of the cylinder wall is $\sim \sigma_{\alpha} / 2$, where $\alpha$ denotes the ion species or $\mathrm{SPC} / \mathrm{E}$ water oxygen atom), are applied. Here, $\epsilon_{c y l}$ is chosen to be $0.3845 \mathrm{~kJ} / \mathrm{mol}$ such that, with the combining rules, the SPC/E water oxygen interacts with the cylinder with a value of $\epsilon_{c y l, o}=0.5 \mathrm{~kJ} / \mathrm{mol}$. Such a choice for $\epsilon_{c y l}$ realizes contact angle values similar to those of real materials for objects of different permittivities. For example, GaAs has a permittivity value greater than 11 and a contact angle of roughly $110^{\circ},[38]$ which is not too different from the contact angle we obtain for a permittivity around this value.[34] However, the water structure about the cylinder was generally insensitive to the power-law form used for this repulsive potential or to the choice of $\epsilon_{c y l}$, as long as the latter was not varied by more than $50 \%$.

In our simulations, the external charges that polarize the solute may be either the partial charges of water or the ion charges. Because we use an explicit solvent, the dielectric permittivity of the volume outside the polarizable solute is taken to be $\varepsilon_{\text {out }}=1$. Note that the electrostatic interaction between the induced charge of the polarizable solutes and an external charge are necessarily attractive since $\varepsilon_{\text {out }}=1 \leq \varepsilon_{\text {in }}$.

Ions were modeled using a combination of Lennard-Jones (L-J) and Coulombic potentials. We have considered only monovalent ions in this study, sampling the Lennard-Jones parameters for three independent models[39-44] of the ionic force fields optimized for the SPC/E water model. Those attributed to Dang, Garrett and Smith (DGS)[39-42] have been widely used in many simulation studies, and were optimized according to the ion-water interaction with values of $\epsilon$ chosen somewhat arbitrarily. Likewise, the $\epsilon$ values of Jensen and Jorgensen (JJ)[43] were also determined somewhat subjectively, but with values of $\sigma$ derived by reproducing the correct ion-water radial distribution functions and hydration free energies in periodic or solvent boundary simulations without using Ewald summation methods. Lastly, Joung and Cheatham (JC)[44] recently defined L-J ion parameters upon optimizing these with respect to achieving the correct hydration free energies as well as the approximate crystal lattice constants and energies of the various monovalent 1:1 salts and ion-water binding distances. The L-J values for these various ion models with respect to SPC/E water are provided in Table I, with ions interacting with each other and with the SPC/E oxygen atom via the usual 12-6 L-J potential

$$
V_{\alpha, \beta}(r)=4 \epsilon_{\alpha, \beta}\left[\left(\frac{\sigma_{\alpha, \beta}}{r_{\alpha, \beta}}\right)^{12}-\left(\frac{\sigma_{\alpha, \beta}}{r_{\alpha, \beta}}\right)^{6}\right]
$$

and with the same combining rules as that of the cylinder $\left[\epsilon_{\alpha, \beta}=\sqrt{\epsilon_{\alpha} \epsilon_{\beta}}\right.$ and $\left.\sigma_{\alpha, \beta}=\left(\sigma_{\alpha}+\sigma_{\beta}\right) / 2\right]$. Each of these models provides relatively similar values of the ion hydration energies[44] (see Table II) albeit using different combinations of $\epsilon$ and $\sigma$. As shown in Table I, the DGS values for both $\epsilon$ and $\sigma$ for the cation and anion are intermediate to those of the JC and JJ models. Compared to the DGS parameters, the JC values have a relatively smaller cation $\sigma$ and larger anion $\sigma$ (in combination with, respectively, larger/smaller $\epsilon$ values to account for roughly the same hydration energies) and the JJ values, the reverse.

Note that both the ions and water models employed in our simulations are non-polarizable. We have used such models to isolate the effects of the solute's polarizability and to avoid results that depend on the polarizable model used for the ion/water force fields. Nonetheless, we have found very similar water structure near the solute upon using a polarizable model. Indeed, other studies have shown that water properties for polarizable and non-polarizable models next to surfaces are fairly independent of solvent polarizability for a wide range of surface permittivity.[45]

Simulations were performed within the canonical ensemble $(N V T)$ at $T=298 \mathrm{~K}$ using the Nosé-Hoover thermostat. In order to maintain the systems at liquid-vapor equilibrium, a liquid-vapor interface, far from the cylindrical solute, was imposed. We note that similar approaches have been considered in other studies.[46, 47] Using such an interface keeps the system at coexistence conditions without having to use a barostat, which may introduce artifacts due to large variations in local pressure at solvent-substrate surfaces. This method also obviates altering the number of the atoms in the NVT simulations, which would be required to maintain similar pressures across different simulations if the interface had not not been used. The dimensions of the simulation box were $160 \times 70 \times 34 \AA^{3}$ with the cylinder's 
axis along the $z$-axis. The solute was completely immersed in water, with the liquid-vapor interface imposed at $x= \pm 50 \AA$. Simulations or this volume required about $7 \times 10^{3}$ water molecules.

The electrostatic interactions between charges, both the external water/ion charges and the induced charges on the polarizable solutes, were calculated using the three-dimensional Ewald summation method with "conducting" boundary conditions. Short-ranged non-Coulombic forces (atomic $r^{-6}$ van der Waals forces) were cut off at a distance of $14 \AA$. A typical simulation consisted of $\sim 10^{5}$ equilibration steps, with a time step of 2 fs, followed by $\sim 10^{6}$ production steps to achieve adequate statistics. Simulations were completed for eight different alkali-halide salts (LiCl, LiI, NaF, NaCl, NaI, KF, KCl, and KI) using the L-J parameters of each of the three models at three different values $\left(\varepsilon_{i n}=1,2\right.$, and 5$)$ of the cylinder permittivity.

\section{RESULTS}

\section{A. Polarizable solutes in pure water}

Simulations were first performed on single cylindrical solutes, with varying degrees of polarizability, completely immersed in pure water. We have considered polarizable solutes with permittivities of $\varepsilon_{i n}=1-5$, which make up a range of values used in predictive macroscopic models of proteins[48-51] and dielectric values of carbon nanoparticles.[52]

Radial density profiles of water oxygen and hydrogen atoms about the solutes are shown in Fig. 1. Increasing the permittivity of the solute results in greater solvation of the solute. For $\varepsilon_{i n}=1$ and 2 , the solute remains perfectly hydrophobic, as observed in long simulations of cylindrical solutes that are initially half immersed in water, i.e., starting with an initial configuration with a planar liquid-vapor interface defined by the long axis of the cylindrical solute and the $\phi=0, \pi$ azimuthal vectors of the cylinder, in which the solute completely de-wets. For larger permittivities, which possess finite contact angles when starting simulations with the cylinder half immersed, a high density contact layer of water forms at the solute surface. This layer resembles the one seen at water-metal interfaces. [53-55] Furthermore, there is greater polar structure of the water near the solute as the permittivity of the solute is increased. Here the water dipoles preferentially align parallel or anti-parallel to the radial vector perpendicular to the solute's surface, as evident in the offset in the hydrogen and oxygen peaks in Fig. 1. This ordering disrupts the hydrogen bonding among the water molecules near the solute, so that the water density does not attain its bulk value until up to $\sim 10$ $\AA$ ( $\approx 3$ water molecular diameters) away from the solute surface for larger values of $\varepsilon_{i n}$. Furthermore, there is an enhancement of the positive (hydrogen) partial charges of the SPC/E water close to the cylinder, which has been observed at interfaces in other explicit water simulations.[56]

\section{B. Salt solutions about hydrophobic solutes}

In the following we investigate the structure of the aqueous solutions next to a hydrophobic substrate with dielectric constant $\varepsilon_{i n}=2$. Computations of the contact angles of this substrate[34] indicate this object is hydrophobic with a contact angle of $180^{\circ}$. We have performed simulations of aqueous solutions at a specific concentration, 1.3 molal, which is in the range relevant for studies of Hofmeister effects in protein solutions and other media[57-60] and which has been utilized in simulation studies of specific ion effects at interfaces.[61, 62] Upon adding salt to the cylinderwater system, a number of features emerge that depend on the type of salt as well as the force fields of the specific ionic model employed. The most apparent of these is the change in the density of the bulk solvent. (Hence, we have therefore kept the bulk molality of the added salt, i.e., the ratio of added salt ions to water molecules, constant in each salt simulation rather than the molarity.) Again, being that the simulations are performed with an explicit vapor-liquid interface, these density changes of the water suggest a great variability of the osmotic coefficients with salt species at vapor-liquid coexistence. As shown in Fig. 2, the density hinges dramatically on the halide anion of the salt, having only a weak dependence on the associated alkali counterion. This reduction of solvent density with increasing anion size, displayed by all three (DGS,JJ,JC) ion models, [63] corresponds to the increase in the osmotic coefficient for larger anions observed in recent simulation studies. [64]

For the sodium ion with L-J parameters defined by the various ionic models, the ion density slowly increase from the cylinder surface, reaching the bulk concentration at $\sim 7-10 \AA$ away from the surface (see Fig. 3). Our results indicate that $\mathrm{Na}^{+}$ions are depleted from the surface. Complete solvation of the ions in the bulk solvent is expected to be energetically favored over the partial solvation that would result if the ions approach the cylinder surface. Moreover, the hydrogen-bonding of the water structure is disrupted by water reorganization about the surface, which may further diminish ion hydration as ions approach the cylinder. We also note that the depletion from the cylinder surface is compatible with the results that would be obtained by considering water as a dielectric continuum. This would be the approach used in most mean field theories that rely on the solution of the Poisson-Boltzmann equation. 
In that case the interaction between $\mathrm{Na}^{+}$and the dielectric should be repulsive, because $\varepsilon_{\text {in }}=2$ would be smaller than the bulk water dielectric constant, and thus $\mathrm{Na}^{+}$would be depleted from the surface. We note however that the physical principle behind the depletion in our simulations is not connected to the image charge repulsion, since in our system this interaction is attractive and small.

Investigation of a smaller cation provides further proof that mean field approaches based on a dielectric continuum are inaccurate near dielectric discontinuities. The lithium ion distributions of $\mathrm{LiCl}$ and LiI, exhibit an initial peak at $\sim 3 \AA$ from the cylinder surface, roughly at the location of the first major oxygen peak of the water profile about the cylinder (see Fig. 4). This is not a trivial result. Lithium has a high hydration free energy, the highest of the three cations investigated here (c.f. Table II). This should result in stronger hydration preventing the ion from approaching the solute interface. From the point of view of mean field theories that neglect the molecular structure of water and model water as a dielectric medium with permittivity $\approx 80, \mathrm{Li}^{+}$should feel a strong image repulsion, and it should be repelled from the surface. If the ion size is considered, this repulsion should be stronger than that of $\mathrm{Na}^{+}$discussed above. Our simulation results show the opposite trend; $\mathrm{Li}^{+}$is observed to approach the solute-water interface moreso than $\mathrm{Na}^{+}$and $\mathrm{K}^{+}$(see supplement[63] for $\mathrm{K}^{+}$distribution) for any of the salts. There is evidence from recent simulations of aqueous solutions that $\mathrm{Li}^{+}$is less repelled than other ions from the water-air interface. [65] This observation is reproduced by all the force fields investigated in this work, although there are quantitative differences across force fields in the height of the first peak next to the solute.

This observation may be rationalized upon analyzing the structure of water about the $\mathrm{Li}^{+}$ion near the interface. First, as compared to the $\mathrm{Na}^{+}$ion, the coordination number of waters about the $\mathrm{Li}^{+}$ion remains relatively unchanged upon comparing the cation-oxygen radial distribution functions (rdf) for ions near the surface and those in the bulk (see Fig. 5). Integrating the first peak of the rdf's (inset of Fig. 5) reveals that the water coordination number for $\mathrm{Li}^{+}$is $N_{C} \approx 4$ for both the surface and bulk ions, whereas the $\mathrm{Na}^{+}$coordination changes from $N_{C} \approx 5.3$ (surface) to $N_{C} \approx 5.6$ (bulk), demonstrating that $\mathrm{Li}^{+}$remains as solvated near the surface of the cylinder as it does in the bulk solvent. (Note that the first peak in the rdf for $\mathrm{Li}^{+}$ions near the surface is even larger than that for ions in the bulk solvent, which suggests that the first solvation shell is more tightly bound for $\mathrm{Li}^{+}$near the surface than in the bulk.) $\mathrm{Li}^{+}$ions can remain solvated near the surface since the water molecules, being four-fold coordinated about the ion both for ions near the solute surface and in bulk water, are arranged tetrahedrally about the $\mathrm{Li}^{+}$ ion (see Fig. 6). This tetrahedral structure, commensurate with the usual tetrahedral structure of water via their hydrogen bonds, sits comfortably at the surface, with only a slight distortion of the tetrahedron at the solute surface and with the "legs" of the tetrahedron adjacent to the surface. This geometrical configuration can be extracted from a distribution plot of the angle, $\theta_{r}$, between the directional vector joining the cation and the oxygen atom of the water molecule and the directional radial vector emanating from the cylinder axis to the location of the cation, i.e., $\cos \left(\theta_{r}\right)=\hat{r}_{\mathrm{C}^{+}-\mathrm{O}} \cdot \hat{r}_{\mathrm{C}^{+}}$(see Fig. 7) Similar distributions are seen for $\mathrm{Li}^{+}$ions near a solute with $\varepsilon_{\text {in }}=1$,[63] suggesting that the concentration of these ions may be enhanced at a generic liquid-vapor interface. The rdf's for the larger $\mathrm{Na}^{+}$ions, however, demonstrate a much larger discrepancy between those ions that lie near the surface and those in the bulk. As shown in Fig. 6, the angle between the vectors of neighboring solvating waters about the $\mathrm{Na}^{+}$ion is $\approx 90^{\circ}$, suggesting a nearly octahedral "bonding" structure. Near the solute surface, a more severe, and hence energetically costly, distortion of the solvation structure at the surface occurs as compared to that of the tetrahedral structure for solvated $\mathrm{Li}^{+}$. As shown in Fig. 7, the three pyramidal "legs" of the octahedral structure adjacent to the solute surface bend away from the surface. Without distortion, the probability would peak at the angle between the body-diagonal, which is defined by the cylindrical inwardly-pointing radial vector normal to the solute surface $\left(\theta_{r}=\pi\right.$ in this geometry) and the face-center vectors along which the octahedral "bonds" are situated. However, one sees this angle is stretched with the peak at roughly $\theta_{r}=0.65 \pi$ rather than at $\left.\theta_{r}=\arccos (-1 / \sqrt{3})\right)$ since the water is pushed away from the solute surface. This distortion in turn reduces the average coordination number of solvating waters about $\mathrm{Na}^{+}$near the surface as compared to the number in the bulk. Hence, as compared to the larger cations, $\mathrm{Li}^{+}$is more readily accommodated at the surface, and, depending on the force field used, it may even prefer to lie nearer the surface than in the bulk (see the peaks shown in Fig. 4).

We find that most ion distributions exhibit the same general features across the different ionic models, but there exist some discrepancies among the specific ion models used. For every salt parametrized by the JJ ion model, the cation distribution grows faster and earlier (with respect to the cylinder surface) than that of its anion counterpart. The cation distributions near the surface for ions using the DGS and JC model parameters, however, may grow faster or slower than that of the accompanying anion depending on the salt species. Fig. 3, for example, shows the accumulated (integrated) radial ionic charge distribution for $\mathrm{NaCl}$ for each of the models, demonstrating this difference. This difference may be attributed, in general, to the lower hydration energies of the cations parametrized with the JJ values than those in which the DGS or JC parameters are employed. Again, ions with greater solvation energies would prefer to be deeper in the bulk solvent, unless, as is the case for $\mathrm{Li}^{+}$, both the ion and its first solvation shell may be contentedly moored near the solute surface.

We also find that a very large disparity among the models for salts containing the iodide anion exists. For each of 
these salts, an initial iodide peak appears near the cylinder surface when the DGS and JC ion parameters are used, whereas no peak is observed for the JJ model parameters as shown in Fig. 8. This may be explained upon considering the slightly smaller hydration energies of the DGS and JC models as compared to that of the JJ model for this ion. However, the differences in these energies are quite small, indicating how extremely sensitive the ion distributions are to the force-field parameters employed. We note that the strong adsorption at the water-cylinder interface is reminiscent of the adsorption discussed by Jungwirth and Tobias,[3] although, according to these authors, the anion polarization was the main driving force for ion adsorption. Our results show that the same positive adsorption is achieved in non-polarizable ion models. These results agree with previous simulations[22-26] and integral equation studies,[66] in which adsorption of larger ions at interfaces using rigid ion models had been observed.

\section{Salt solutions about polarizable solutes}

In the following we address the influence of solute polarizability on the ion adsorption. We consider here solutes with a permittivity of $\varepsilon_{i n}=5$. This permittivity results in contact angles of about $130^{\circ}$,[34] i.e., changing the permittivity of the cylinder from $\varepsilon_{i n}=2$ to $\varepsilon_{i n}=5$ results in partial wetting of the formerly perfectly hydrophobic cylinder. The increased interaction of the cylinder with the water molecules is accompanied by greater cylinder-ion interactions. Thus, the strong sensitivity of the ionic distributions on the ion force-field model employed is further enhanced upon increasing the permittivity of the solute.

As for the solutes with $\varepsilon_{i n}=2$, the ion distributions varied significantly by ion species, as well as by the ion forcefield model employed, for the more polarizable solutes. Whereas the cation concentration near the solute dominated that of the anion one for the JJ model as compared to the DGS and JC models for the hydrophobic cylinder, for the $\varepsilon_{i n}=5$ cylinder, as shown, e.g., for sodium salts in Fig. 9, there is a greater concentration of the cation near the solute for the DGS and JC models than for the JJ model. Here, the effective size of the ion becomes more important than its hydration energy. The smaller (on average) hydration energy of the cations of the JJ model with respect to the other ion models resulted in the cation concentrations next to the hydrophobic $\left(\varepsilon_{\text {in }}=2\right)$ cylinder being larger than that for their anion counterparts. This ionic property of the JJ model is now eclipsed by the cations' larger sizes (see Table I) upon determining their distributions about the more polarizable cylinder. The smaller-sized cations of the DGS and JC models allow them to approach the cylinder more closely and, thus, to interact more strongly with their attractive image charges. For these models, this results in a strong physisorption of the cations at the cylinder surface. Although the same occurs for the JJ model, it is much weaker. Nevertheless, for every model and for each salt, the cation concentration near the cylinder surface exceeds that of the anion.

The concentration of cations near the cylinder surface, however, is not completely determined by the ion's size. As shown in Fig. 10, the distribution of sodium ions $\left(\mathrm{Na}^{+}\right)$is more heavily peaked at the cylinder surface (for both the DGS and JC models) than either the smaller $\mathrm{Li}^{+}$or the larger $\mathrm{K}^{+}$ion. This non-monotonicity in the distribution may be attributed to the subtle competition between the ion's size and its hydration energy. For example, although the $\mathrm{Li}^{+}$ion is smaller, and thus may interact more strongly with its attractive image charge reflected in the solute, its large hydration energy in the bulk liquid must be overcome when it approaches the solute surface. Figures 11-13 show the radial distribution functions and water distributions of the solvation shell about ions adsorbed at the solute surface. For these adsorbed ions, there is a decrease in the water coordination number, but this loss in solvation energy is compensated by the attractive interaction of the ion with the image charges of the solute. Note that this reduction in the coordination number, however, does not dramatically affect the geometrical structure (type of "bonding") of the solvation water about the adsorbed ions, other than to eliminate one (or two) of the "bonding" water molecules.

The cation concentration at the cylinder surface also influences that of their anion counterparts. The strong $\mathrm{Na}^{+}$ peak at the surface attracts anions, engendering an anion peak as well, although this depends on the anion species. Figure 14 shows that the iodide ion, which, again, has the smallest hydration energy of the anion species tested here, is most heavily attracted to the solute surface, followed by the chloride ion. However, in the DGS model, for example, the smaller peak concentrations of the other cations $\left(\mathrm{Li}^{+}\right.$and $\left.\mathrm{K}^{+}\right)$are not large enough to induce the accumulation of chloride ions at the solute surface (see Fig. 14). Overall we find that the adsorption increases with anion size, where the small ion, $F^{-}$, shows depletion from the surface, whereas $I^{-}$shows adsorption. Again, these results cannot be explained considering the image forces arising from a dielectric discontinuity where water is modeled as a dielectric continuum, or using a theory that relies on ion valency only. Yet, again, these anion distributions depend very much on the ion force-field model employed; whereas anion peaks may develop for some cation-anion pairs in the DGS and JC models, no anion accumulation at the surface ever occurs for the JJ model (see Fig. 6 of the supplement[63]). 


\section{CONCLUSIONS AND FINAL REMARKS}

In this paper we have investigated, using computer simulations, aqueous electrolyte solutions next to cylindrical hydrophobic objects in which the degree of their polarizability was defined via their dielectric permittivity. This situation is relevant to a number of solutes, e.g., DNA, nanorods and protein bundles. At the salt concentrations used here $(1.3 \mathrm{~m})$ the ionic interactions are expected to be significantly screened. We have explicitly modeled the dielectric discontinuity arising from the different permittivity of the nanoscopic solute and the aqueous solution. With this purpose we have employed a computational algorithm we have introduced recently. In this way we take a step beyond mean-field approaches by including discrete solvent effects explicitly. Also, the simple image-charge model for the solute takes into account the collective many-body effects of the interactions of every atom/molecule with its and other atom's/molecule's image in the solute arising from the dielectric response of the solute, which is infeasible upon using a mean-field theory for the solute-solvent partical dispersion interaction. Present work has initially demonstrated that mean-field theories cannot capture the effect this collective interaction has on the solvent structure near the solute. Moreover we have discussed the dependence of ion adsorption on the ion-water-solute force field.

At hydrophobic solute surfaces the ion distributions depend on a number of factors: (i) the ion solvation energy, since ions with smaller solvation energies may be found nearer the cylinder since they are less inclined as those with larger solvation energies to be in the bulk solvent; (ii) the water structure about the cylinder and the corresponding role it has on solvation; (iii) the image charge interactions arising from the dielectric discontinuities associated with the water-solute interface; and (iv) the choice of the cylinder and ion potentials, where the distance of closest approach to the cylinder depends on the size $(\sigma)$ of the ion.

The solvation energy is commonly used in the literature to explain the depletion of ions from interfaces. Similarly image charge interactions have been used before in the Onsager-Samaras theory to explain the same effect. Mean field theories model these interactions for a system consisting of two dielectric media of different permittivity. According to this, because the permittivity of the solute is smaller than that of water one would expect depletion of cations (or anions) from the interface, irrespective of the nature of the cation (or anion). We note that neither of these ideas, solvation or image forces, can explain the behavior of the smallest cation we have investigated here, $\mathrm{Li}^{+}$. Despite having a larger solvation energy we find that $\mathrm{Li}^{+}$exhibits adsorption at a perfectly hydrophobic surface . Because we are not including frequency-dependent dispersion interactions, we conclude that this is a water mediated effect. Note that the $\mathrm{Li}^{+}$adsorption peak at the interface may be overlooked in studies of, e.g., the distribution of ions at the solvent-vapor interface due to fluctuations of the interface (capillary waves),[67] which are suppressed next to the solute surface. Studies of ions at the solvent-vapor interface are currently underway in which we account for interfacial fluctuations.

Similarly we find strong adsorption of iodide at hydrophobic surfaces, although in this instance this adsorption is fairly sensitive to the force-field employed. We find that two popular force fields, Dang, Garrett and Smith[39-42] and Joung and Cheatham[44] predict adsorption, whereas the Jensen and Jorgensen force field[43] predicts depletion. We find that these trends are independent of the counterion $\left(\mathrm{Li}^{+}, \mathrm{Na}^{+}, \mathrm{K}^{+}\right)$considered. This result indicates that selectivity due to ion adsorption may be a subtle effect, hence care has to be exercised in the modeling of these effects through modification of the dispersion interactions. We also note that the iodide adsorption we observe follows the observation reported first by Jungwirth and Tobias and which has recently been a popular subject of discussion in the literature. Nonetheless, in agreement with more recent computations[22-26] it appears that ion polarization is not needed to produce ion adsorption at hydrophobic interfaces, i.e., adsorption is also observed using rigid, nonpolarizable ion models.

We have further investigated the influence of solute polarizability on ion adsorption. Upon increasing the permittivity of the solute from $\varepsilon_{i n}=2$ to $\varepsilon_{i n}=5$, the cation interaction with the solute begins to dominate that of the anion due to the average smaller size of the cations. This, of course, depends on the ion force-field model employed. However, the competition between an ion's size and its hydration energy provides some subtle difference in the ion distributions near the solute surface, namely the non-monotonicity observed in the concentration amplitude with cation size. Furthermore, the anion distributions are also affected by their respective cations, depending very much on the anion model employed. We qualify that the strong cationic adsorption at the solute-solvent interface may be a remnant of the image-charge model used for the solute, since such a model becomes inaccurate at the interface. A more rigorous, quantum mechanical, study would need to be performed to determine how the electronic structure of the solute and solvent particles influence the adsorption, but this goes beyond the scope of the present paper. However, we believe this is a quantitative issue being that the same method was used for all force-fields employed in this study and that trends similar to our results would be expected in real systems.

We find ion selectivity cannot be accounted for by approaches in which the solvent is modeled as a dielectric continuum. We also find that ion adsorption can be strongly dependent on the force field investigated.Granted, the ion force fields used have not been paremeterized with respect to the ions' properties at an interface, and may be too "soft" upon approaching the solute surface, but we are limited to those 
force fields that are available and hope to have shown the adsorption dependence of the different force-field models (which may disqualify certain force-field models upon comparing these results to real systems). Nonetheless, the results for ion adsorption should be considered when developing theoretical approaches to explain ion effects, e.g., those ion-specific effects responsible for the Hofmeister series. We find strong evidence that the adsorption characteristics of the ions are determined by the water solvation around the ions. Hence the description of dielectric discontinuities in aqueous solutions requires the explicit consideration of the solvent. We note that mean field models that include water as a dielectric continuum to model dielectric discontinuities, would predict ion desorption on the basis of a repulsive image interaction. Our results show this notion is incorrect both for cations and for anions. Overall, our results indicate that modeling interfaces by considering water as a dielectric continuum may provide incorrect trends for ion selectivity.

Finally, solute polarizability is shown to have a strong effect on the adsorption of ions on neutral solutes. These effects must be properly accounted for in order to develop a full description of the interactions between ions and surfaces.

\section{Acknowledgments}

AW would like to thank the Alexander von Humboldt Foundation and FB The Royal Society and The Leverhulme Trust for financial support. The simulations were performed using computation facilities provided by the Imperial College High Performance Computing Service and the Institute of Theoretical Physics II at Heinrich Heine University Düsseldorf.

[1] V. Bergeron, Forces and structure in thin liquid soap films, J. Phys.: Condens. Matter 11, R215 (1999).

[2] A. A. Kornyshev, D. J. Lee, S. Leikin, and A. Wynveen, Structure and interactions of biological helices, Rev. Mod. Phys. 79, 943 (2007).

[3] P. Jungwirth and D. J. Tobias, Specific ion effects at the air/water interface, Chem. Rev. 106, 1259 (2006).

[4] P. B. Petersen and R. J. Saykally, On the nature of ions at the liquid water surface, Annu. Rev. Phys. Chem. 57, 333 (2006).

[5] B. Derjaguin and L. Landau, Theory of the stability of strongly charged lyophobic sols and of the adhesion of strongly charged particles in solutions of electrolytes, Acta Physico Chemica URSS 14, 633 (1941).

[6] E. J. W. Verwey and J. T. G. Overbeek, Theory of the stability of lyophobic colloids (Elsevier, Amsterdam, 1948).

[7] J. Faraudo and F. Bresme, Anomalous dielectric behavior of water in ionic newton black films, Phys. Rev. Lett. 92, 236102 (2004).

[8] F. Bresme and J. Faraudo, Computer simulation studies of newton black films, Langmuir 12, 5127 (2004).

[9] J. Faraudo and F. Bresme, Origin of the short-range, strong repulsive force between ionic surfactant layers, Phys. Rev. Lett. 94, 077802 (2005).

[10] S. Leikin, V. A. Parsegian, and D. C. Rau, Hydration forces, Annu. Rev. Phys. Chem. 44, 369 (1993).

[11] J. Israelachvili and H. Wennerstrom, Role of hydration and water structure in biological and colloidal interactions, Nature 379, 219 (1996).

[12] S. Marcelja, Hydration in electrical double layers, Nature 385, 689 (1997).

[13] S. Marcelja and N. Radic, Repulsion of interfaces due to boundary water, Chem. Phys. Lett. 42, 129 (1976).

[14] S. Leikin and A. A. Kornyshev, Theory of hydration forces - nonlocal electrostatic interaction of neutral surfaces, J. Chem. Phys. 92, 6890 (1990).

[15] N. A. M. Besseling, Theory of hydration forces between surfaces, Langmuir 13, 2113 (1997).

[16] M. Boström, D. R. M. Williams, and B. W. Ninham, Specific ion effects: Why DLVO theory fails for biology and colloid systems, Phys. Rev. Lett. 87, 168103 (2001).

[17] W. Kunz, P. Lo Nostro, and B. W. Ninham, The present state of affairs with Hofmeister effects, Curr. Opin. Coilloid In. 9, 1 (2004).

[18] F. Hofmeister, Zur Lehre von der Wirkung der Salze, Arch. Exp. Pathol. Pharmakol 24, 247 (1888).

[19] L. Onsager and N. N. T. Samaras, The surface tension of Debye-Huckel electrolytes, J. Chem. Phys. 2, 528 (1934).

[20] Y. Levin, Polarizable ions at interfaces, Phys. Rev. Lett. 102, 147803 (2009).

[21] J. Abascal, F. Bresme, and P. Turq, The influence of concentration and ionic-strength on the cluster structure of highlycharged electrolyte solutions, Mol. Phys. 81, 143 (1994).

[22] B. Schnell, R. Schurhammer, and G. Wipff, Distribution of hydrophobic ions and their counterions at an aqueous liquidliquid interface: A molecular dynamics investigation, J. Phys. Chem. B 108, 2285 (2004).

[23] F. Bresme, M. Gonzalez-Melchor, and J. Alejandre, Influence of ion size asymmetry on the properties of ionic liquid-vapour interfaces, J. Phys.: Condens. Matter 17, S3301 (2005). 
[24] S. Pal and F. Müller-Plathe, Molecular dynamics simulation of aqueous NaF and NaI solutions near a hydrophobic surface, J. Phys. Chem. B 109, 6405 (2005).

[25] B. Eggimann and J. Siepmann, Size effects on the solvation of anions at the aqueous liquid-vapor interface, J. Phys. Chem. C 112, 210 (2008).

[26] D. J. V. A. dos Santos, F. Müller-Plathe, and V. C. Weiss, Consistency of ion adsorption and excess surface tension in molecular dynamics simulations of aqueous salt solutions, J. Phys. Chem. C 112, 19431 (2008).

[27] R. Kjellander and S. Marcelja, Electrolyte solutions between uncharged walls, Chem. Phys. Lett. 142, 485 (1987).

[28] E. Ruckenstein and M. Manciu, Specific ion effects via ion hydration: II. Double layer interaction, Adv. Colloid Interfac. 105, 177 (2003).

[29] L. Boinovich and A. Emelyanenko, The image-charge forces in thin films of solutions with non-polar solvent, Adv. Colloid Interfac. 104, 93 (2003).

[30] E. Wernersson and R. Kjellander, On the effect of image charges and ion-wall dispersion forces on electric double layer interactions, J. Chem. Phys. 125, 154702 (2006).

[31] M. M. Hatlo and L. Lue, The role of image charges in the interactions between colloidal particles, Soft Matter 4 , 1582 (2008).

[32] V. Ballenegger and J.-P. Hansen, Dielectric permittivity profiles of confined polar fluids, J. Chem. Phys. 122, 114711 (2005).

[33] A. Wynveen and F. Bresme, Interactions of polarizable media in water: A molecular dynamics approach, J. Chem. Phys. 124, $104502(2006)$.

[34] F. Bresme and A. Wynveen, On the influence of solute polarizability on the hydrophobic interaction, J. Chem. Phys. 126, 044501 (2007).

[35] F. Bresme and A. Wynveen, Interactions of Polarizable Media in Water and the Hydrophobic Interaction in "Aspects of Physical Biology: Biological Water, Protein Solutions and Replication", edited by G. Franzese and M. Rubi (SpringerVerlag, Berlin Heidelberg, 2008), vol. 752 of Lect. Notes Phys., pp. 43-62.

[36] J. Alejandre, G. A. Chapela, F. Bresme, and J.-P. Hansen, The short range anion-H interaction is the driving force for crystal formation of ions in water, J. Chem. Phys. 130, 174505 (2009).

[37] R. Allen, J.-P. Hansen, and S. Melchionna, Electrostatic potential inside ionic solution confined by dielectrics: a variational approach, Phys. Chem. Chem. Phys. 3, 4177 (2001).

[38] K. Autumn, M. Sitti, Y. A. Liang, A. M. Peattie, W. R. Hansen, S. Sponberg, T. W. Kenny, R. Fearing, J. N. Israelachvili, and R. J. Full, Evidence for van der waals adhesion in gecko setae, Proc. Nat. Acad. Sci. USA 17, 12252 (2002).

[39] L. X. Dang, Development of nonadditive intermolecular potentials using molecular-dynamics - solvation of Li+ and F-ions in polarizable water, J. Chem. Phys. 96, 6970 (1992).

[40] L. X. Dang and B. C. Garrett, Photoelectron-spectra of the hydrated iodine anion from molecular-dynamics simulations, J. Chem. Phys. 99, 2972 (1993).

[41] D. E. Smith and L. X. Dang, Computer-simulations of $\mathrm{NaCl}$ association in polarizable water, J. Chem. Phys. 100, 3757 (1994).

[42] L. X. Dang, Mechanism and thermodynamics of ion selectivity in aqueous-solutions of 18-crown-6 ether - A moleculardynamics study, J. Am. Chem. Soc. 117, 6954 (1995).

[43] K. P. Jensen and W. L. Jorgensen, Halide, ammonium, and alkali metal ion parameters for modeling aqueous solutions, J. Chem. Theory Comput. 2, 1499 (2006).

[44] I. S. Joung and T. E. Cheatham III, Determination of alkali and halide monovalent ion parameters for use in explicitly solvated biomolecular simulations, J. Phys. Chem. B 112, 9020 (2008).

[45] I.-C. Yeh and M. L. Berkowitz, Effects of the polarizability and water density constraint on the structure of water near charged surfaces: Molecular dynamics simulations, J. Chem. Phys. 112, 10491 (2000).

[46] L. G. Camara and F. Bresme, Molecular dynamics simulations of crystallization under confinement at triple point conditions, J. Chem. Phys. 119, 2792 (2003).

[47] X. H. Huang, R. H. Zhou, and B. J. Berne, Drying and hydrophobic collapse of paraffin plates, J. Phys. Chem. B 109, $3546(2005)$.

[48] A. Warshel, S. T. Russel, and A. K. Churg, Macroscopic models for studies of electrostatic interactions in proteins: Limitations and applicability, Proc. Nat. Acad. Sci. USA 81, 4785 (1984).

[49] G. King, F. S. Lee, and A. Warshel, Microscopic simulations of macroscopic dielectric-constants of solvated proteins, J. Chem. Phys. 95, 4366 (1991).

[50] C. N. Schutz and A. Warshel, What are the dielectric "constants" of proteins and how to validate electrostatic models?, Proteins 44, 400 (2001).

[51] J. W. Pitera, M. Falta, and W. F. van Gunsteren, Dielectric properties of proteins from simulation: The effects of solvent, ligands, pH, and temperature, Biophys. J. 80, 2546 (2001).

[52] D. Lu, Y. Li, U. Ravaioli, and K. Schulten, Empirical nanotube model for biological applications, J. Phys. Chem. B 109, 11461 (2005).

[53] A. Kohlmeyer, W. Witschel, and E. Spohr, Molecular dynamics simulations of water/metal and water/vacuum interfaces with a polarizable water model, Chem. Phys. 213, 211 (1996).

[54] E. Spohr, Molecular simulation of the electrochemical double layer, Electrochim. Acta 213, 1697 (1999).

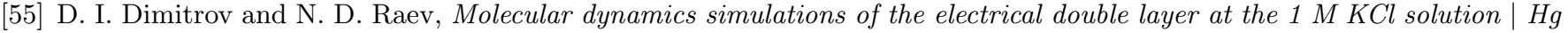
electrode interface, J. Electroanal. Chem. 486, 1 (2000).

[56] J. Dzubiella and J.-P. Hansen, Competition of hydrophobic and coulombic interactions between nanosized solutes, J. Chem. Phys. 121, 5514 (2004). 
[57] T. Arakawa and S. N. Timasheff, Preferential interactions of proteins with salts in concentrated solutions, Biochemistry 21, 6525 (1982).

[58] M. G. Cacace, E. M. Landau, and J. J. Ramsden, The Hofmeister series: salt and solvent effects on interfacial phenomena, Q. Rev. Biophys. 30, 241 (1997).

[59] E. Kokkoli and C. F. Zukoski, Interactions between hydrophobic self-assembled monolayers. Effects of salt and the chemical potential of water on adhesion, Langmuir 14, 1189 (1998).

[60] J. W. Kurutz and S. H. Xu, Hofmeister solute effects on hydrophobic adhesion forces in SFM experiments, Langmuir 17, 7323 (2001).

[61] E. Spohr, Some recent trends in computer simulations of aqueous double layers, Electrochim. Acta 49, 23 (2003).

[62] R. Zangi, M. Hagen, and B. J. Berne, Effect of ions on the hydrophobic interaction between two plates, J. Am. Chem. Soc. 129, 4678 (2007).

63] See Supplementary Material Document No. xxxx for additional results.

[64] I. Kalcher and J. Dzubiella, Structure-thermodynamics relation of electrolyte solutions, J. Chem. Phys. 130, 134507 (2009).

[65] D. Horinek, A. Herz, L. Vrbka, F. Sedlmeier, S. Mamatkulov, and R. R. Netz, Specific ion adsorption at the air/water interface: The role of hydrophobic solvation, Chem. Phys. Lett. 479, 173 (2009).

[66] M. Kinoshita, Structure of aqueous electrolyte solutions near a hydrophobic surface, Condens. Matter Phys. 10, 387 (2007).

[67] F. Bresme, P. T. E. Chacón, and K. Tay, Intrinsic structure of hydrophobic surfaces: The oil-water interface, Phys. Rev. Lett. 101, 056102 (2008). 
TABLE I: Lennard Jones parameters from Dang, Garrett, and Smith;[39-42] Joung and Cheatham;[44] and Jensen and Jorgensen,[43] adapted for the combining rules of $\sigma$ and $\epsilon$ and water models used in these studies. [44]

\begin{tabular}{lllllll}
\hline Ion & $(\mathrm{DGS})$ & \multicolumn{3}{c}{$(\mathrm{JC})$} & $(\mathrm{JJ})$ & \\
& $\sigma$ & $\epsilon$ & $\sigma$ & $\epsilon$ & $\sigma$ \\
& $(\AA)$ & $(\mathrm{kJ} / \mathrm{mol})$ & $(\AA)$ & $(\mathrm{kJ} / \mathrm{mol})$ & $(\AA)$ & $(\mathrm{kJ} / \mathrm{mol})$ \\
\hline $\mathrm{Li}+$ & 1.506 & 0.6904 & 1.4094 & 1.4089 & 2.8633 & 0.00209 \\
$\mathrm{Na}+$ & 2.35 & 0.54392 & 2.1595 & 1.4755 & 4.0116 & 0.00209 \\
$\mathrm{~K}+$ & 3.332 & 0.4184 & 2.8384 & 1.7979 & 4.9221 & 0.00209 \\
$\mathrm{~F}-$ & 3.168 & 0.8368 & 4.0215 & 0.0310 & 3.0491 & 2.9706 \\
$\mathrm{Cl}-$ & 4.401 & 0.4184 & 4.8305 & 0.0535 & 3.9675 & 2.9706 \\
$\mathrm{I}-$ & 5.167 & 0.4184 & 5.2011 & 0.1790 & 4.6358 & 2.9706 \\
\hline
\end{tabular}

TABLE II: Hydration free energies (in $\mathrm{kJ} / \mathrm{mol}$ ) for the three different ion models in SPC/E water (from Ref. [44]).

\begin{tabular}{llll}
\hline Ion & $($ DGS $)$ & $(\mathrm{JC})$ & $(\mathrm{JJ})$ \\
\hline $\mathrm{Li}+$ & 475.7 & 474.0 & 441.8 \\
$\mathrm{Na}+$ & 364.8 & 369.9 & 319.7 \\
$\mathrm{~K}+$ & 273.2 & 297.1 & 248.9 \\
$\mathrm{~F}-$ & 518.0 & 501.2 & 500.4 \\
$\mathrm{Cl}-$ & 367.4 & 373.6 & 380.7 \\
$\mathrm{I}-$ & 300.4 & 311.3 & 324.3 \\
\hline
\end{tabular}

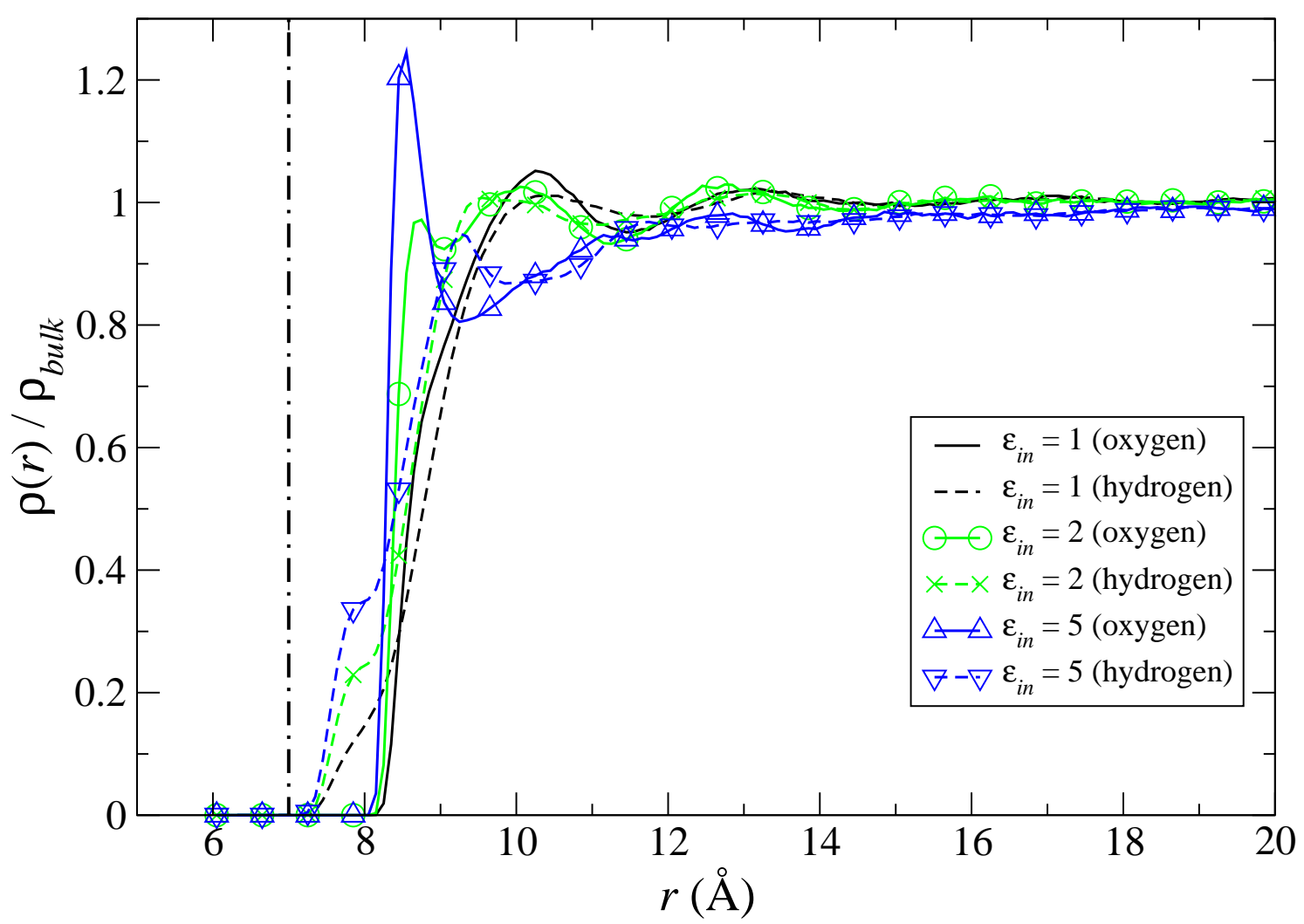

FIG. 1: Oxygen and hydrogen radial density profiles around the cylindrical solute as a function of solute permittivity. The vertical dash-dot line in this plot, and all following plots, shows the location of the cylindrical solute hard wall, which is also the location of the image charge plane $\left(R_{c}=7 \AA\right)$. 


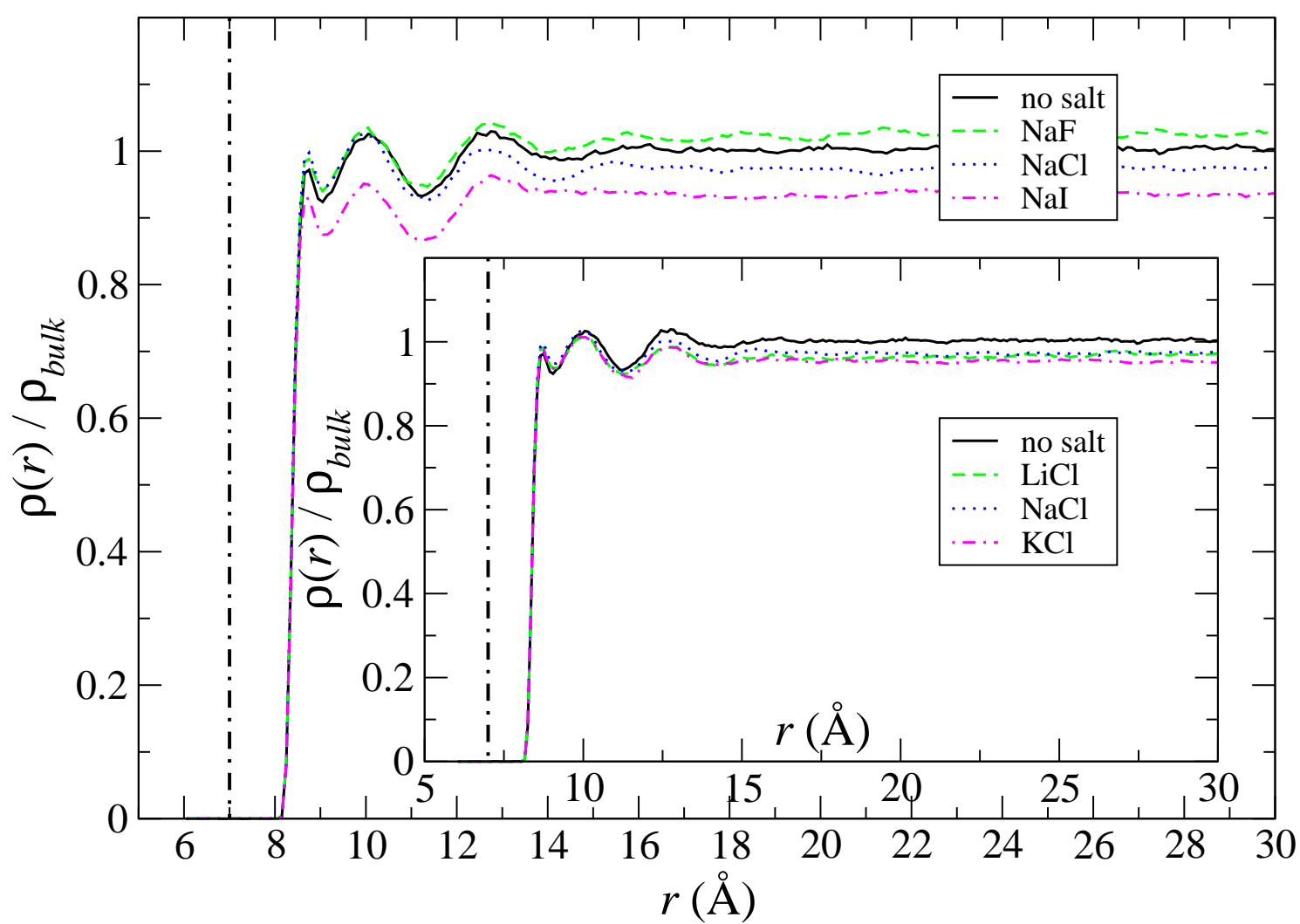

FIG. 2: Oxygen radial density profiles around a cylindrical solute of $\varepsilon_{i n}=2$ for sodium-anion salts with different anion counterparts (Inset: cation-chloride salts with different cations) for the DGS ion models. 


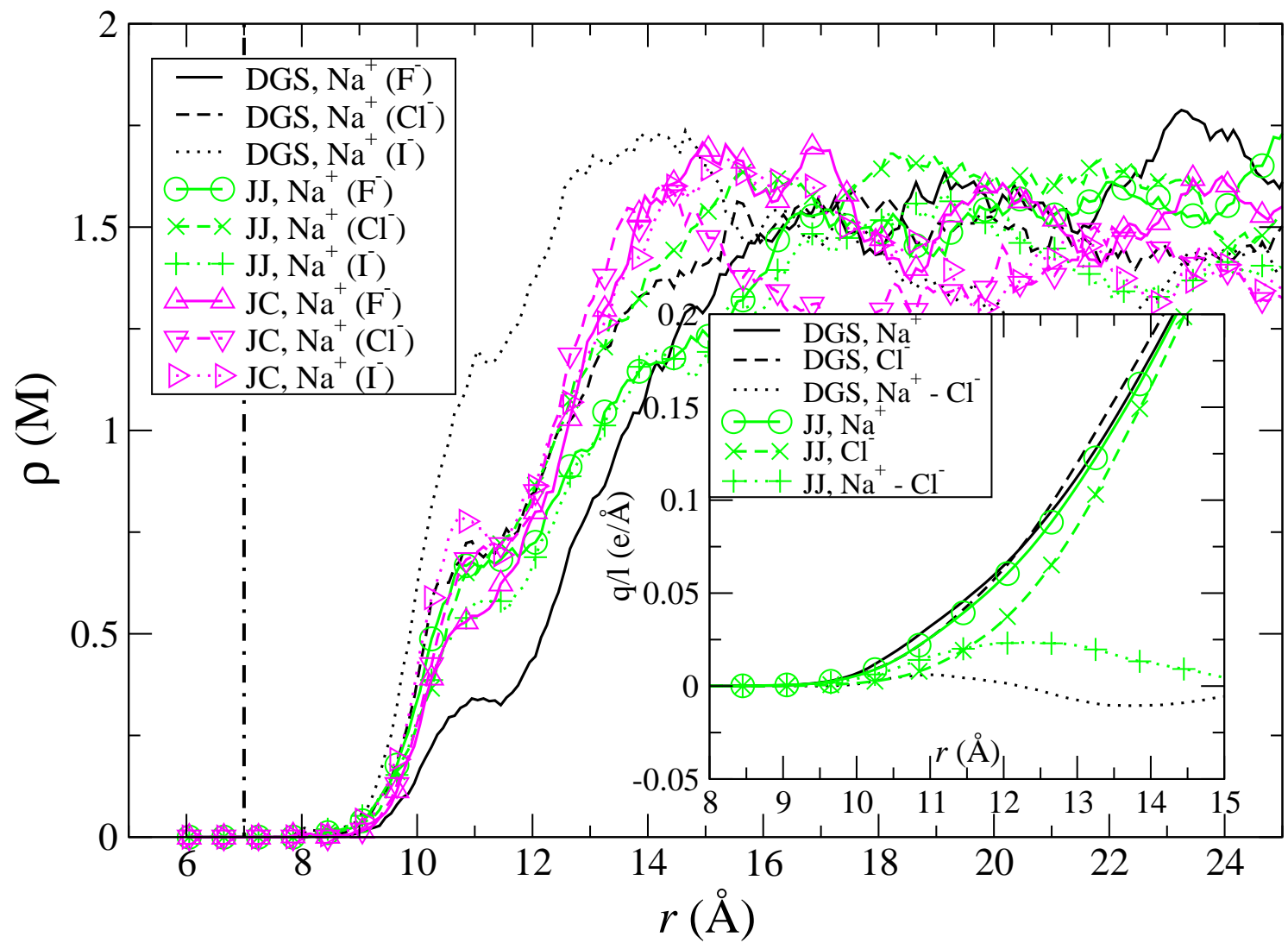

FIG. 3: Sodium radial density profiles around a cylindrical solute of $\varepsilon_{i n}=2$ for sodium-anion salts with different anion counterparts for the three ion models. (Inset) Cumulative (integrated) $\mathrm{Na}^{+}$and $\mathrm{Cl}^{-}$radial ion density and their difference showing the dependence on the ion models. (Note that the salt ion concentrations in this and subsequent figures are defined in terms of molarity, rather than molality, so that the number of ions in a given volume can be easily worked out in these systems. Again, the bulk water density is found to change when varying salt species.) 


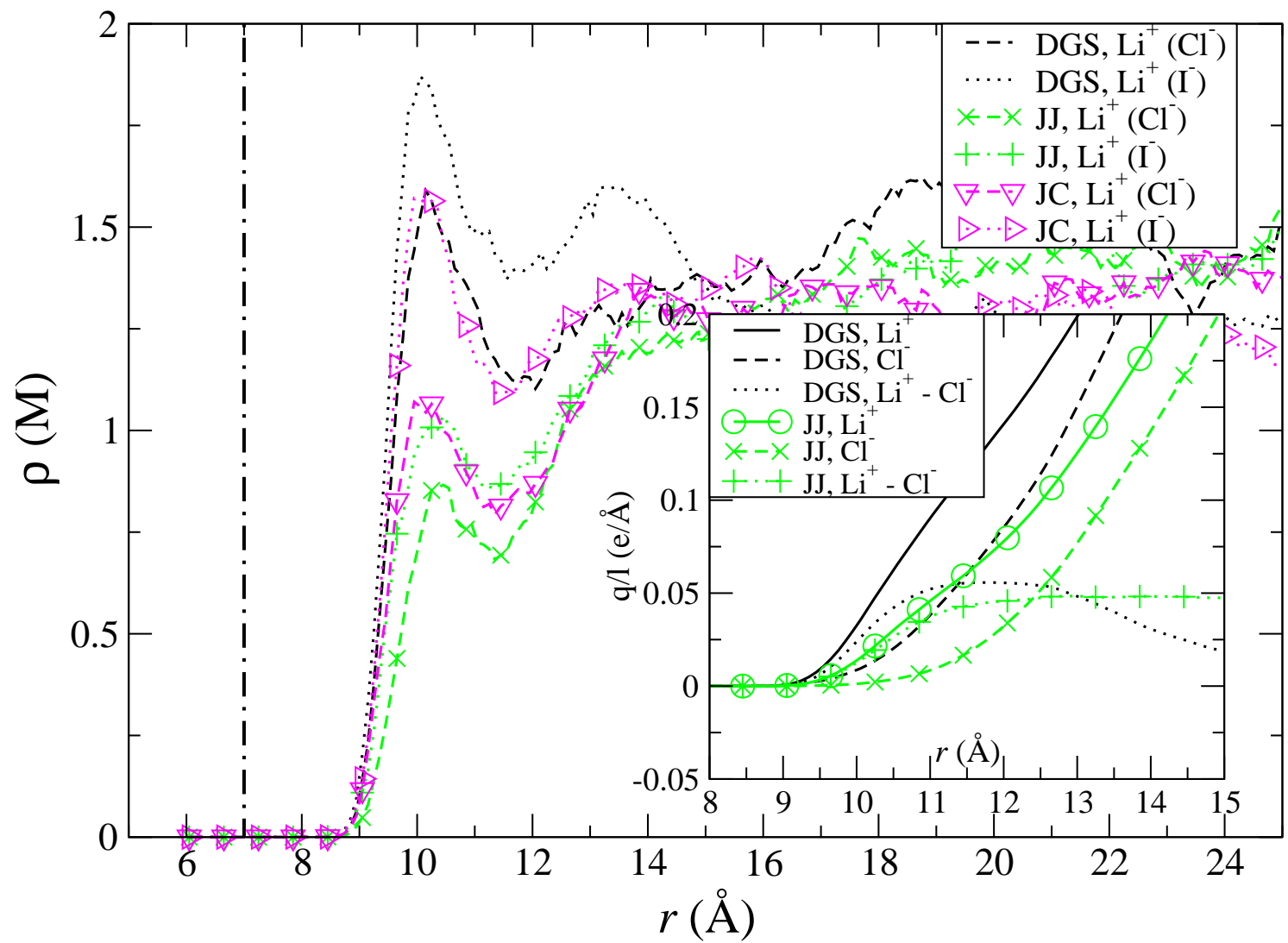

FIG. 4: Lithium radial density profiles around a cylindrical solute of $\varepsilon_{i n}=2$ for lithium-anion salts with different anion counterparts for the three ion models. (Inset) Cumulative (integrated) $\mathrm{Li}^{+}$and $\mathrm{Cl}^{-}$radial ion densities and their difference showing the dependence on the ion models. 


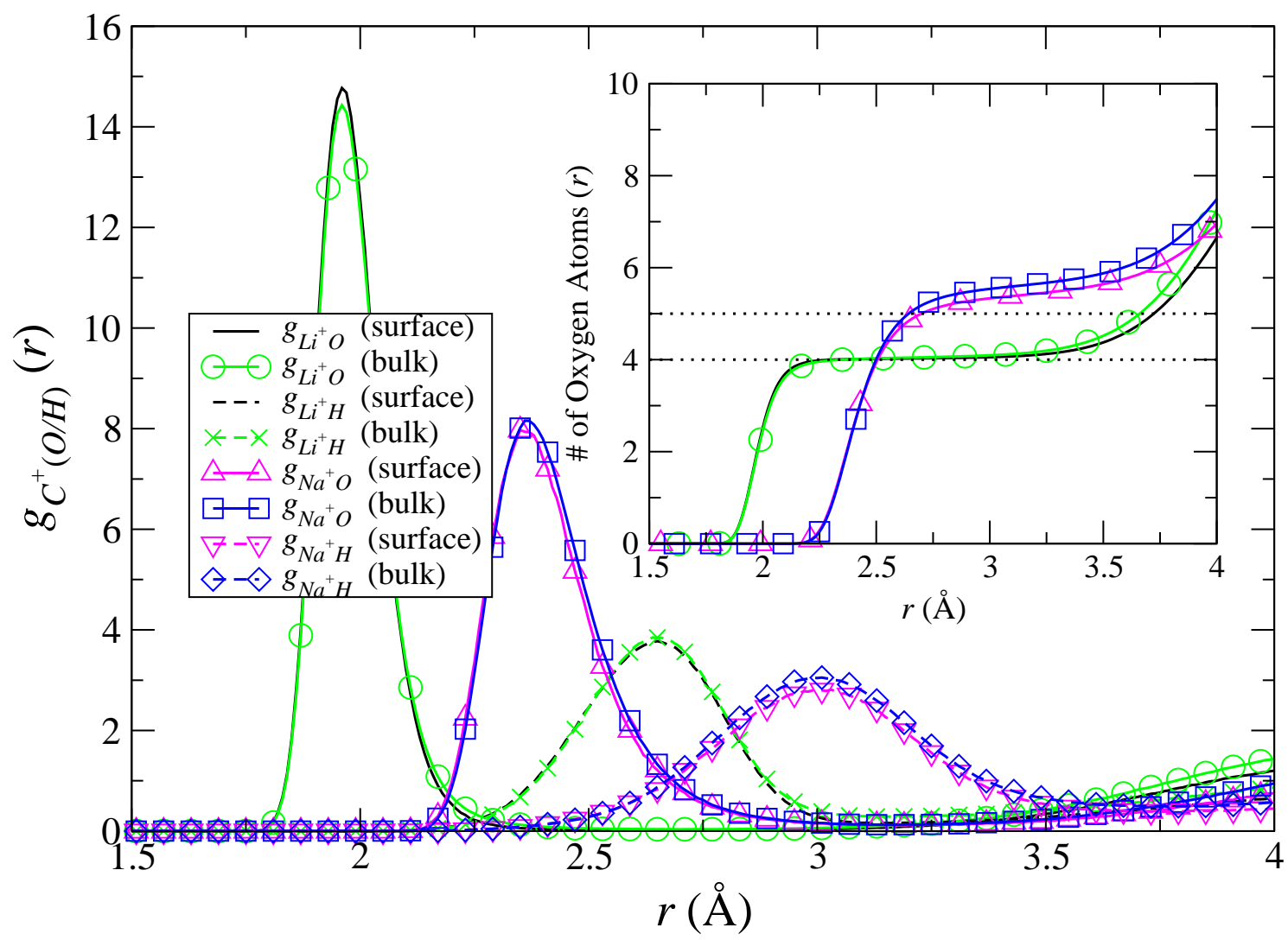

FIG. 5: The radial distribution functions of water about the $\mathrm{Li}^{+}$and $\mathrm{Na}^{+}$ions in (DGS) $\mathrm{LiCl}$ and $\mathrm{NaCl}$ salt simulations of a cylindrical solute of $\varepsilon_{i n}=2$. Curves labeled "surface" correspond to the rdf's of ions within $\approx 3 \AA$ of the solute surface, which accounts for those ions within the peak $(r<11 \AA)$ of the $\mathrm{Li}^{+}$distribution shown in Fig. 4. Note the slightly larger $\mathrm{Li}^{+}-\mathrm{O}$ peak for ions near the surface. (Inset) The integrated rdf, which reveals the coordination number of water molecules in the ions' first solvation shells. 


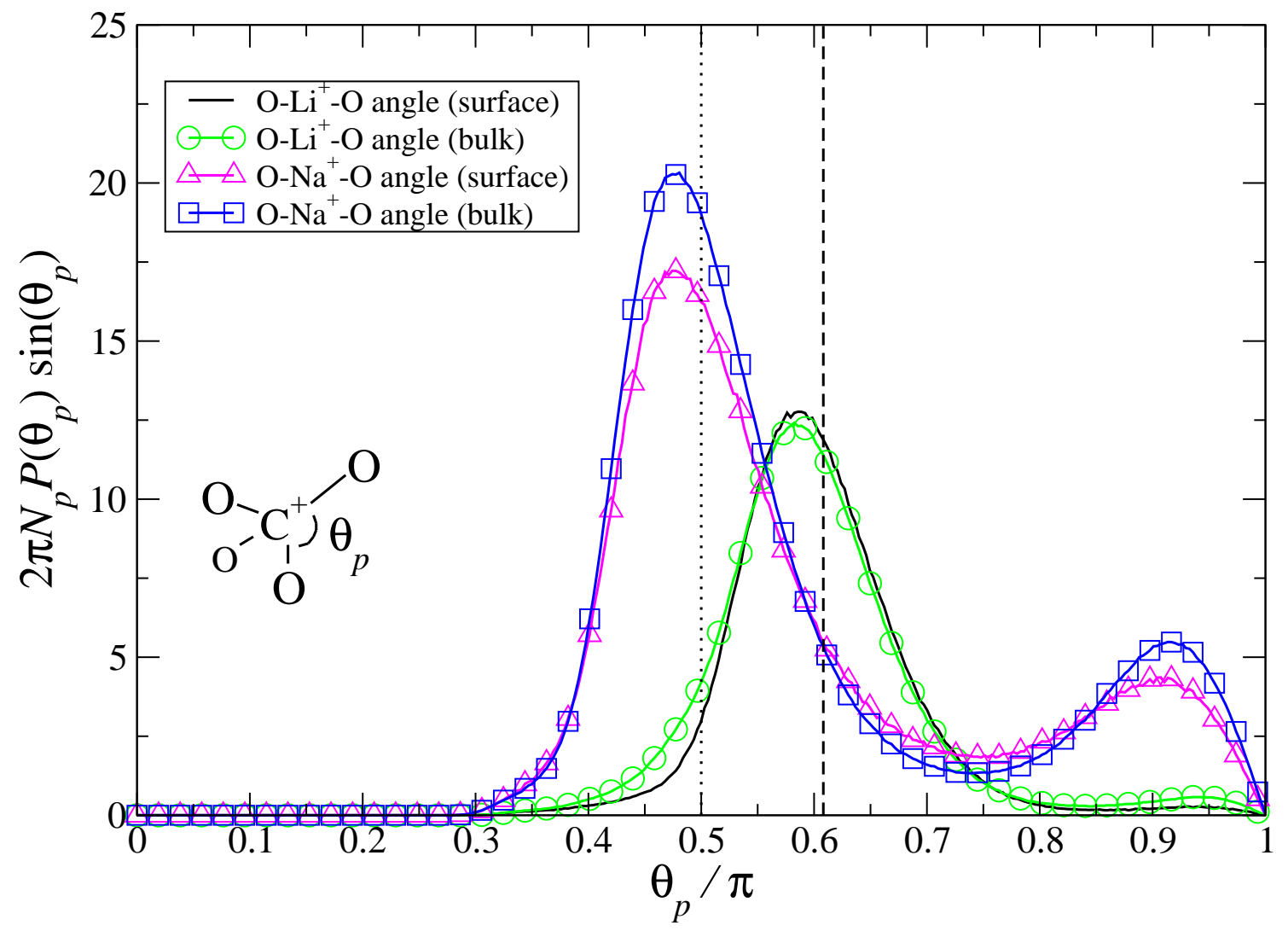

FIG. 6: The probability distribution, such that $\int P\left(\theta_{P}\right) d \Omega=1$ for each of the O-C $\mathrm{C}_{-} \mathrm{O}$ "bond" pair angles, of the "bond" angle defined by the two vectors connecting the cation and two of the water oxygen atoms in the first solvation shell for (DGS) $\mathrm{LiCl}$ and $\mathrm{NaCl}$ salt simulations. The dashed line corresponds to the tetrahedral angle $\left(\arccos (-1 / 3)=109.5^{\circ}\right)$ and the dotted line to the octahedral angle $\left(90^{\circ}\right) . N_{p}$ corresponds to the number of distinct $\mathrm{O}_{-} \mathrm{C}^{+}-\mathrm{O}$ configurations of the water oxygen atoms within the first solvation shell, e.g., for four-fold water coordination about the cation, $N_{p}=6$. 


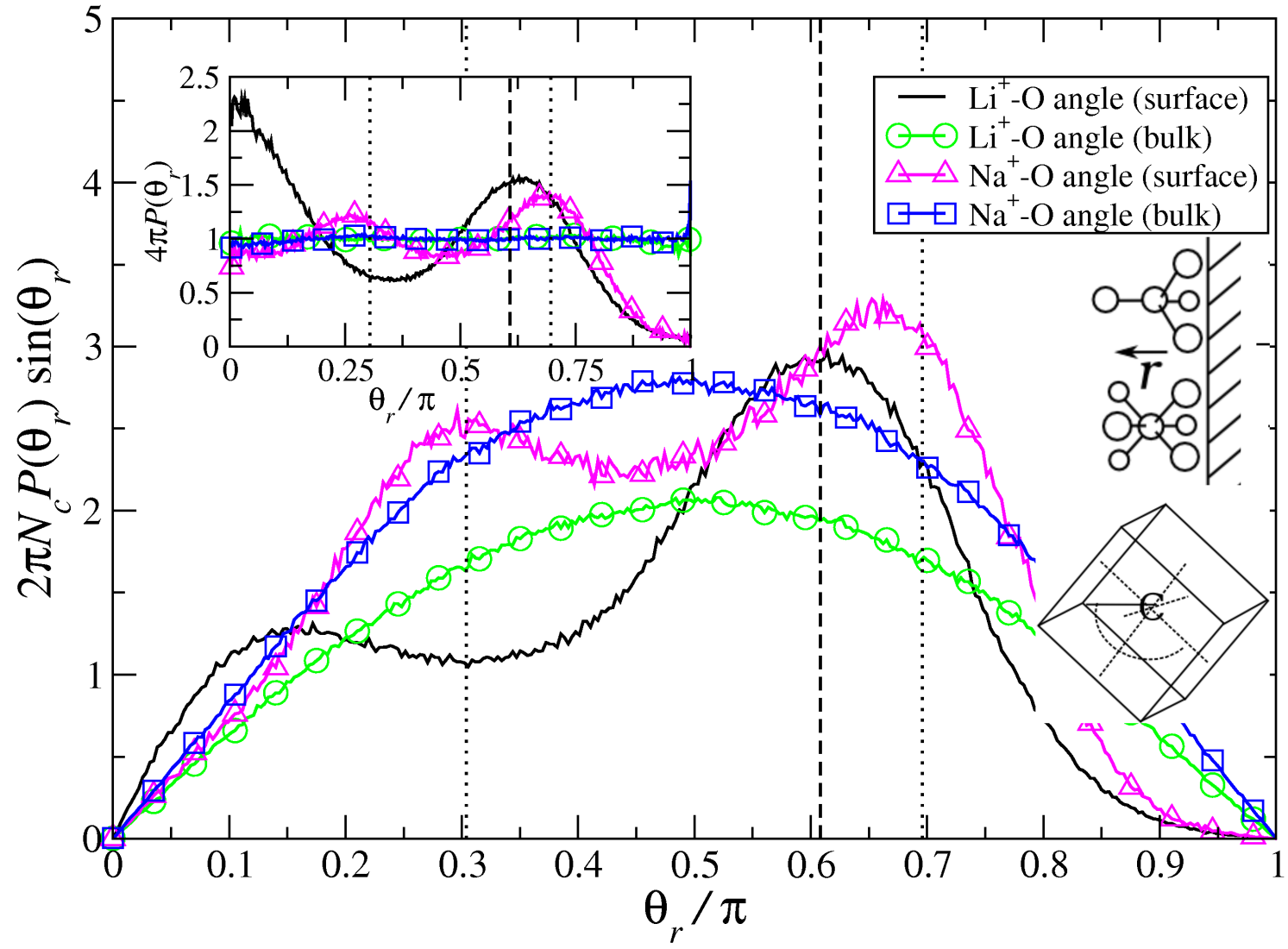

FIG. 7: The normalized probability distribution, without the solid angle correction, of the angle between the vector connecting the cation and a water oxygen atom in the first solvation shell and the radial vector from the solute axis to the cation location for (DGS) $\mathrm{LiCl}$ and $\mathrm{NaCl}$ salt simulations. The dashed line corresponds to the tetrahedral angle $\left(\arccos (-1 / 3)=109.5^{\circ}\right)$ and the dotted lines to the angle $(\arccos ( \pm(1 / \sqrt{3})))$ between the face-centered bond vectors and the corner (or opposite corner) of a cube defined by the octahedral bonding structure as diagrammed in the figure. $N_{c}$ corresponds to the coordination number of water molecules about the cation. (Inset) The solid-angle corrected distribution which takes into account the polar-angle weighting. 


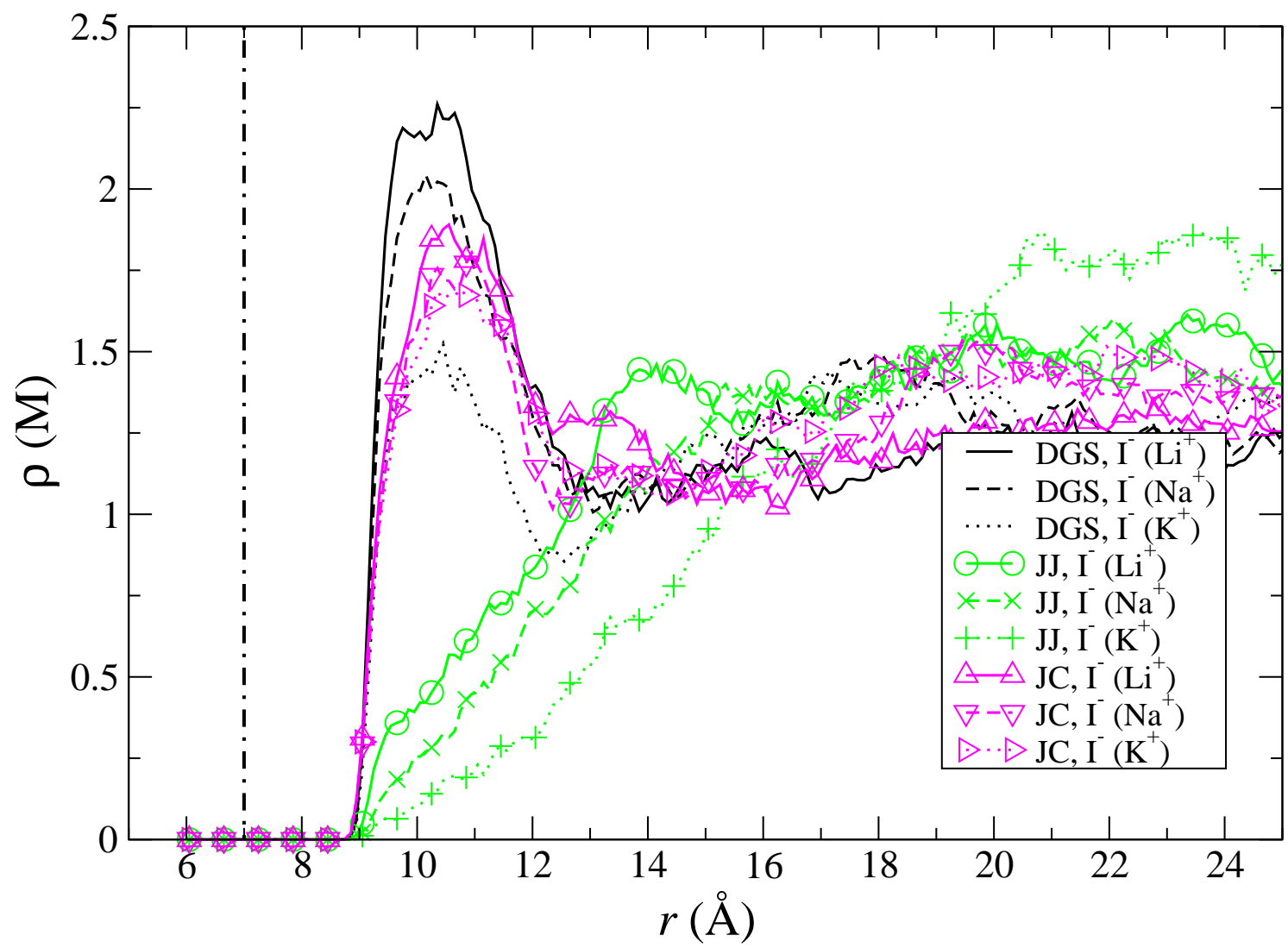

FIG. 8: Iodide radial density profiles around a cylindrical solute of $\varepsilon_{i n}=2$ for cation-iodide salts with different cation counterparts for the three ion models. 


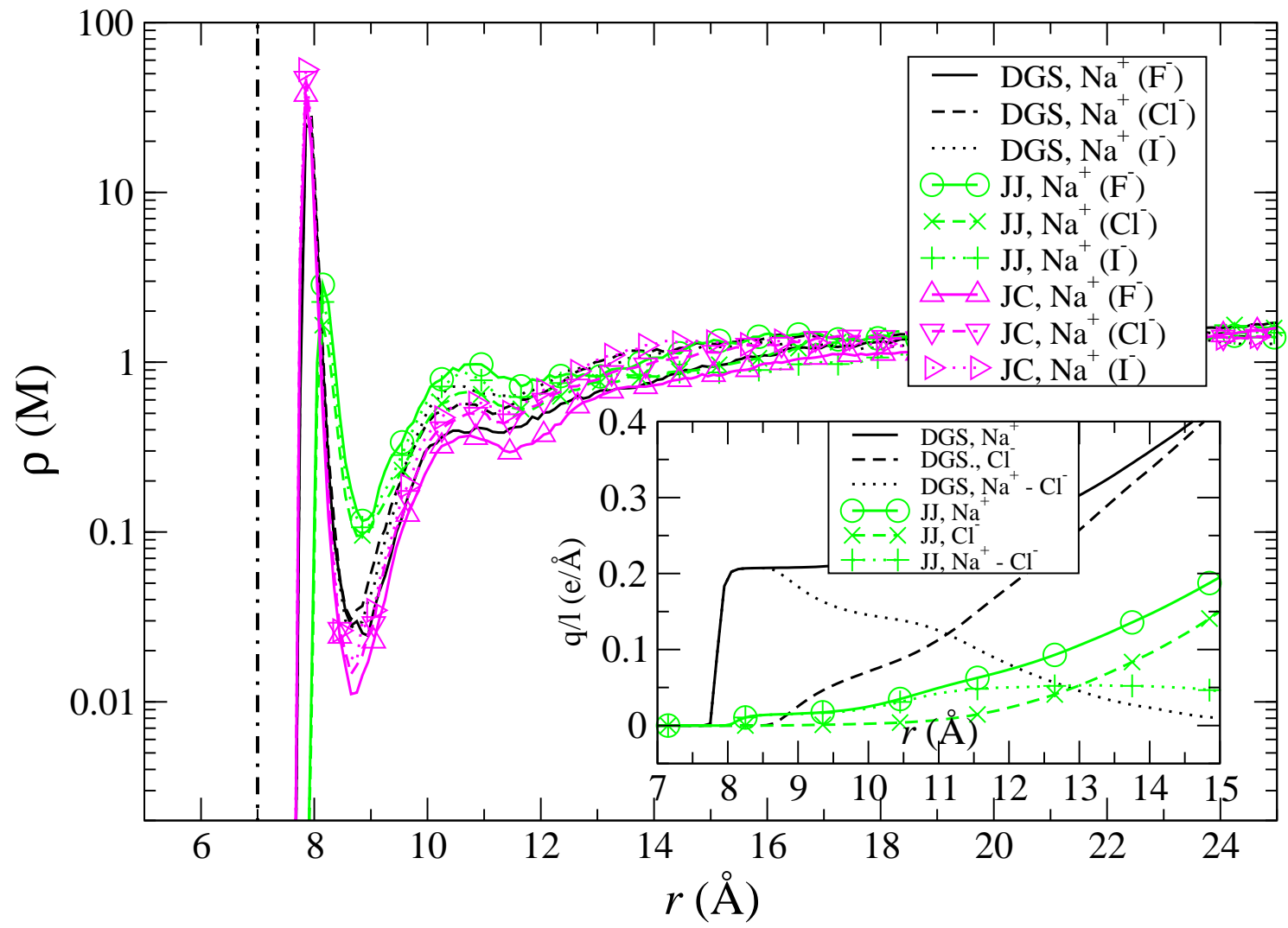

FIG. 9: Sodium radial density profiles around a cylindrical solute of $\varepsilon_{i n}=5$ for sodium-anion salts with different anion counterparts for the three ion models. (Inset) Cumulative (integrated) $\mathrm{Na}^{+}$and $\mathrm{Cl}^{-}$radial ion densities and their difference showing the dependence on the ion models. 


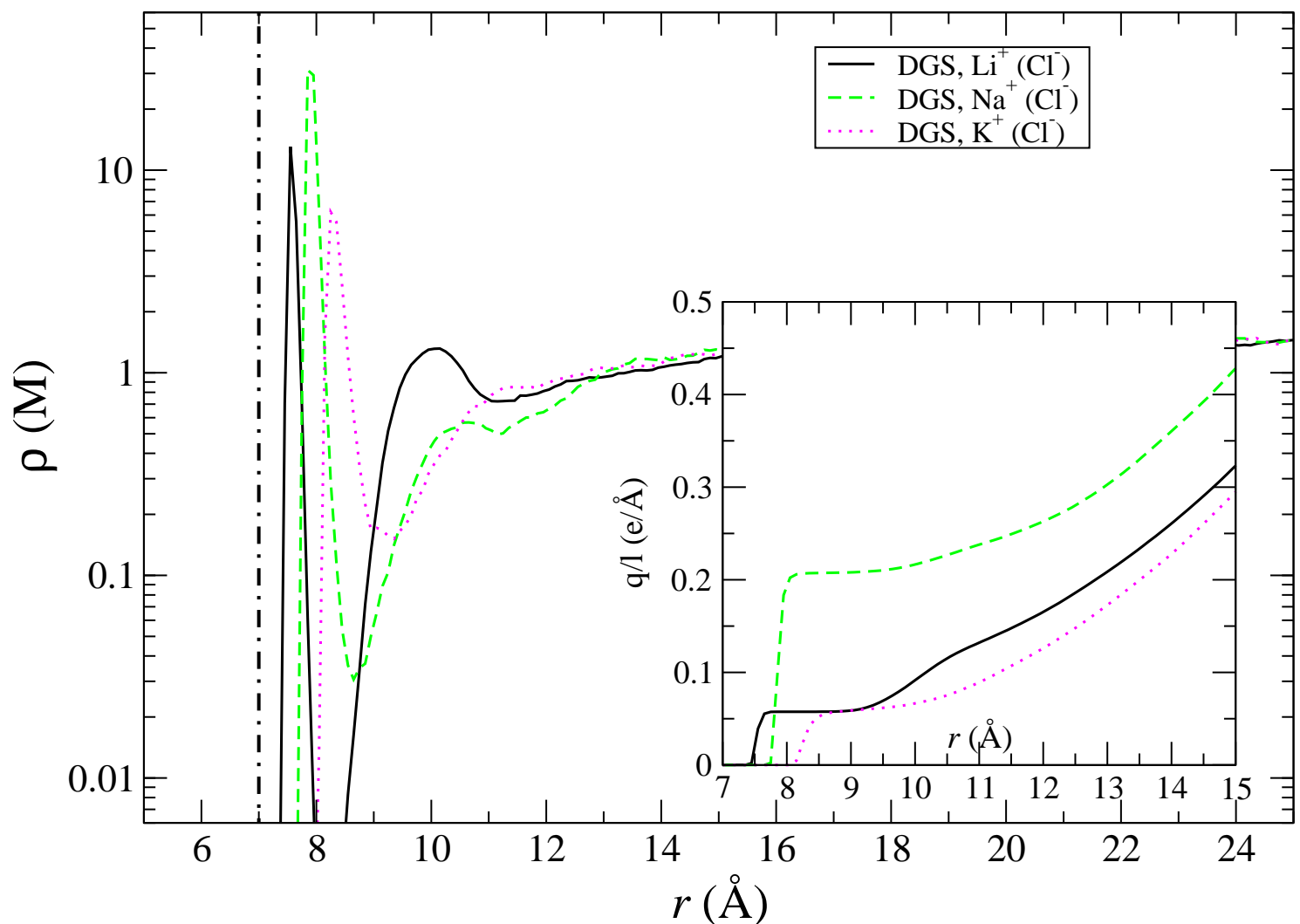

FIG. 10: Radial density profiles of the cations around a cylindrical solute of $\varepsilon_{i n}=5$ for cation-chloride salts. (Inset) The corresponding cumulative (integrated) radial ion densities. 


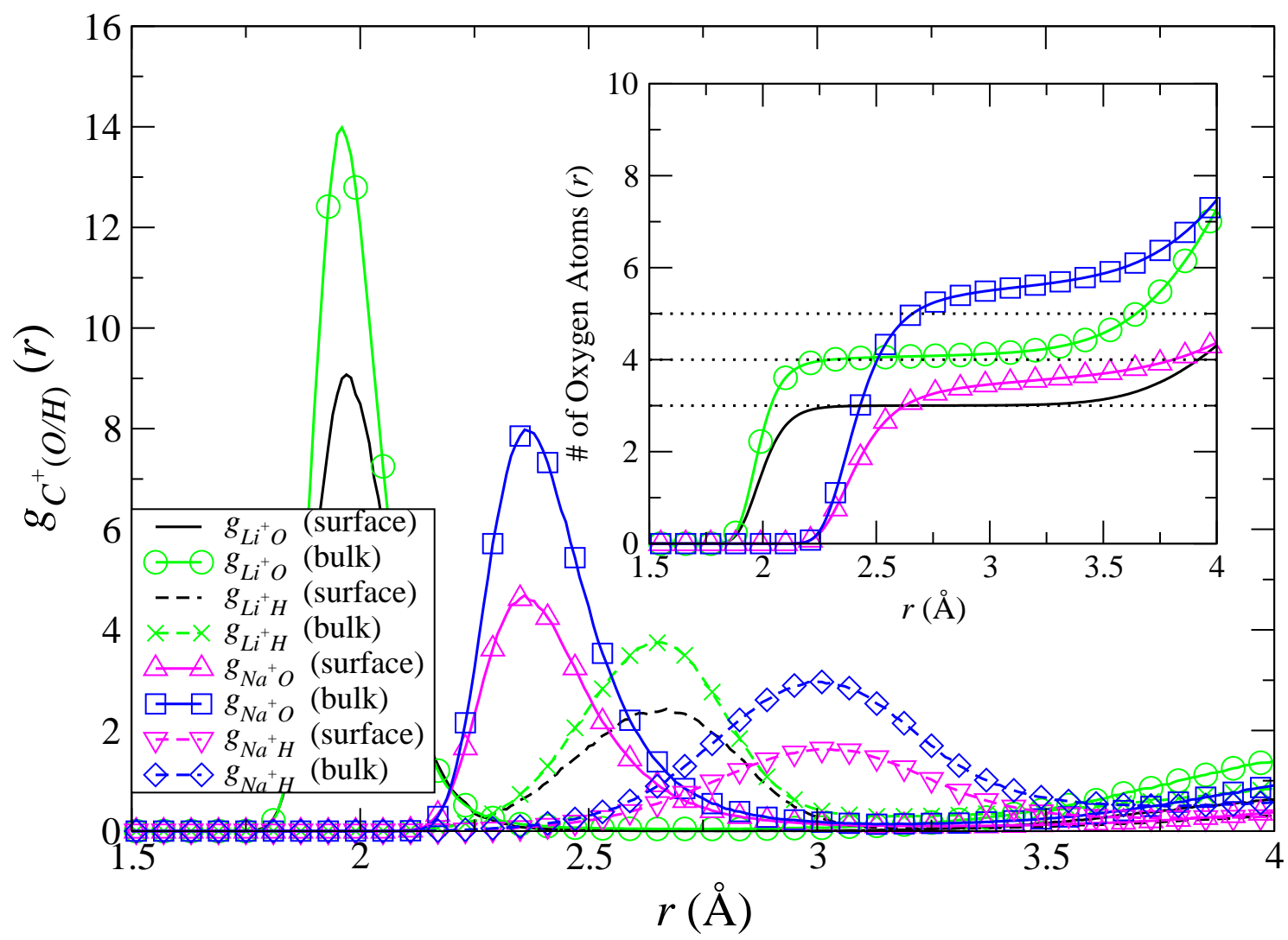

FIG. 11: The radial distribution functions of water about the $\mathrm{Li}^{+}$and $\mathrm{Na}^{+}$ions in (DGS) $\mathrm{LiCl}$ and $\mathrm{NaCl}$ salt simulations of a cylindrical solute of $\varepsilon_{i n}=5$. Curves labeled "surface" correspond to the rdf's of ions within $\approx 1 \AA$ of the solute surface, which accounts for those adsorbed ions within the peak $(r<9 \AA)$ of the cation distribution shown in Fig. 10. (Inset) The integrated rdf, which reveals the reduction of the coordination number of water molecules in the ions' first solvation shells for ions near the solute surface as compared to bulk. Here, the $\mathrm{Li}^{+}$coordination number is reduced by one water molecule, whereas that for $\mathrm{Na}^{+}$is reduced by two. 


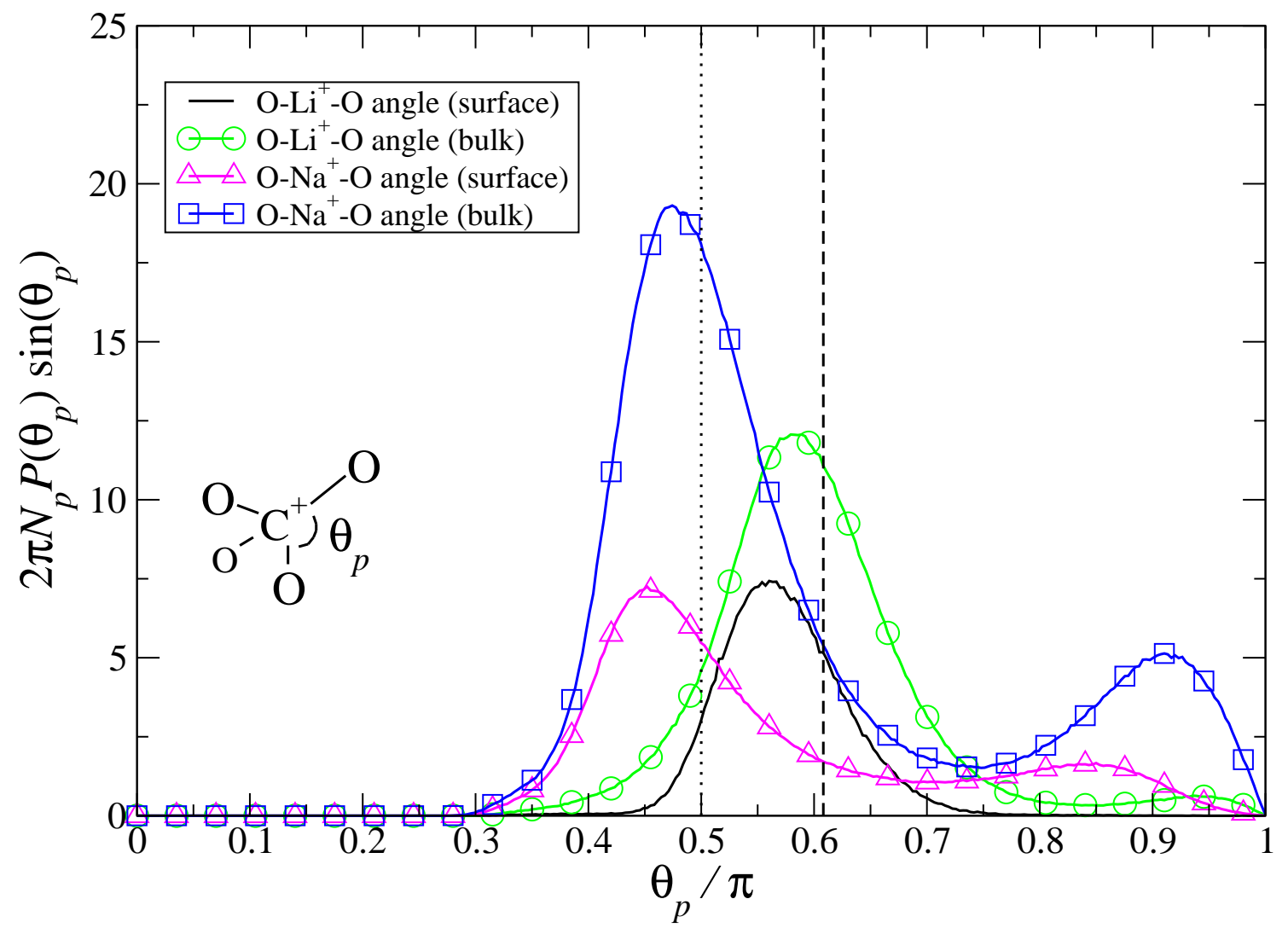

FIG. 12: The probability distribution of the "bond" angle defined by the vectors connecting the cation and two of the water oxygen atoms in the first solvation shell for (DGS) $\mathrm{LiCl}$ and $\mathrm{NaCl}$ salt simulations. The dashed line corresponds to the tetrahedral angle $\left(\arccos (-1 / 3)=109.5^{\circ}\right)$ and the dotted line to the octahedral angle $\left(90^{\circ}\right)$. The bonding configurations for both adsorbed $\mathrm{Li}^{+}$and $\mathrm{Na}^{+}$remain relatively unchanged as compared to the $\varepsilon_{\text {in }}=2$ case, with $\mathrm{Li}^{+}$having a tetrahedral-like arrangement within its solvation shell and $\mathrm{Na}^{+}$having a slightly distorted octahedral arrangement. (See Fig. 13.) 


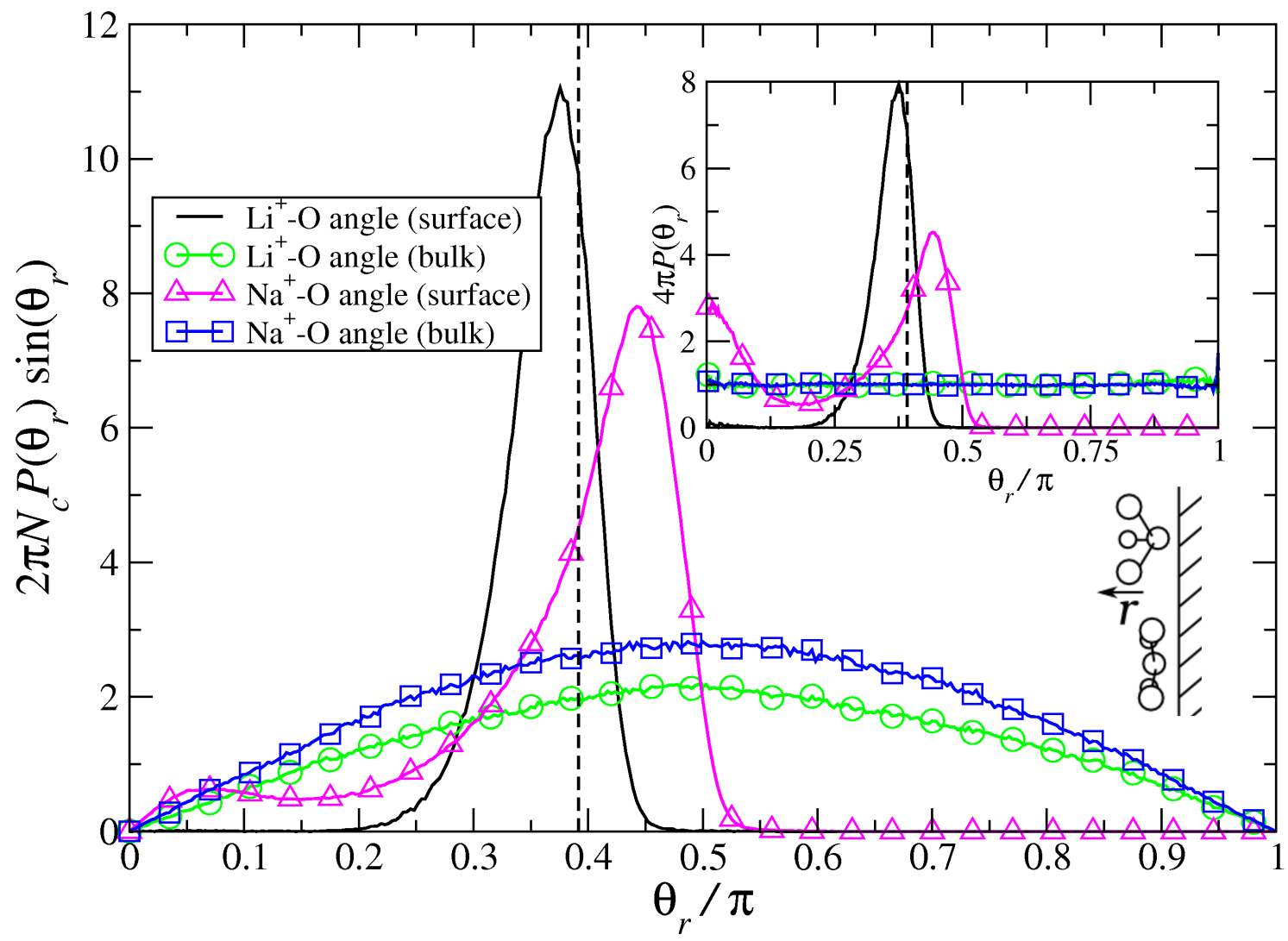

FIG. 13: The probability distribution of the angle between the vector connecting the cation and a water oxygen atom in the first solvation shell and the radial vector from the solute axis to the cation location for (DGS) $\mathrm{LiCl}$ and $\mathrm{NaCl}$ salt, $\varepsilon_{\text {in }}=$ 5 simulations. The dashed line corresponds to the the supplement of the tetrahedral angle $\left(180^{\circ}-\arccos (-1 / 3)=70.5^{\circ}\right)$. According to these distributions and those shown in the previous figure (Fig. 12), the water bonding structure may be extraced and is given by the diagram within this figure. Here, as opposed to the $\varepsilon_{i n}=2$ case, the legs of the tetrahedron of the $\mathrm{Li}^{+}$ point away from the solute surface, providing a three-fold coordination for $\mathrm{Li}^{+}$. Likewise, the distributions suggest a distorted (away from the surface) square planar structure for the larger $\mathrm{Na}^{+}$ion, with a coordination number between three and four (see Fig. 11). (Inset) The solid-angle corrected distribution which takes into account the polar-angle weighting. 


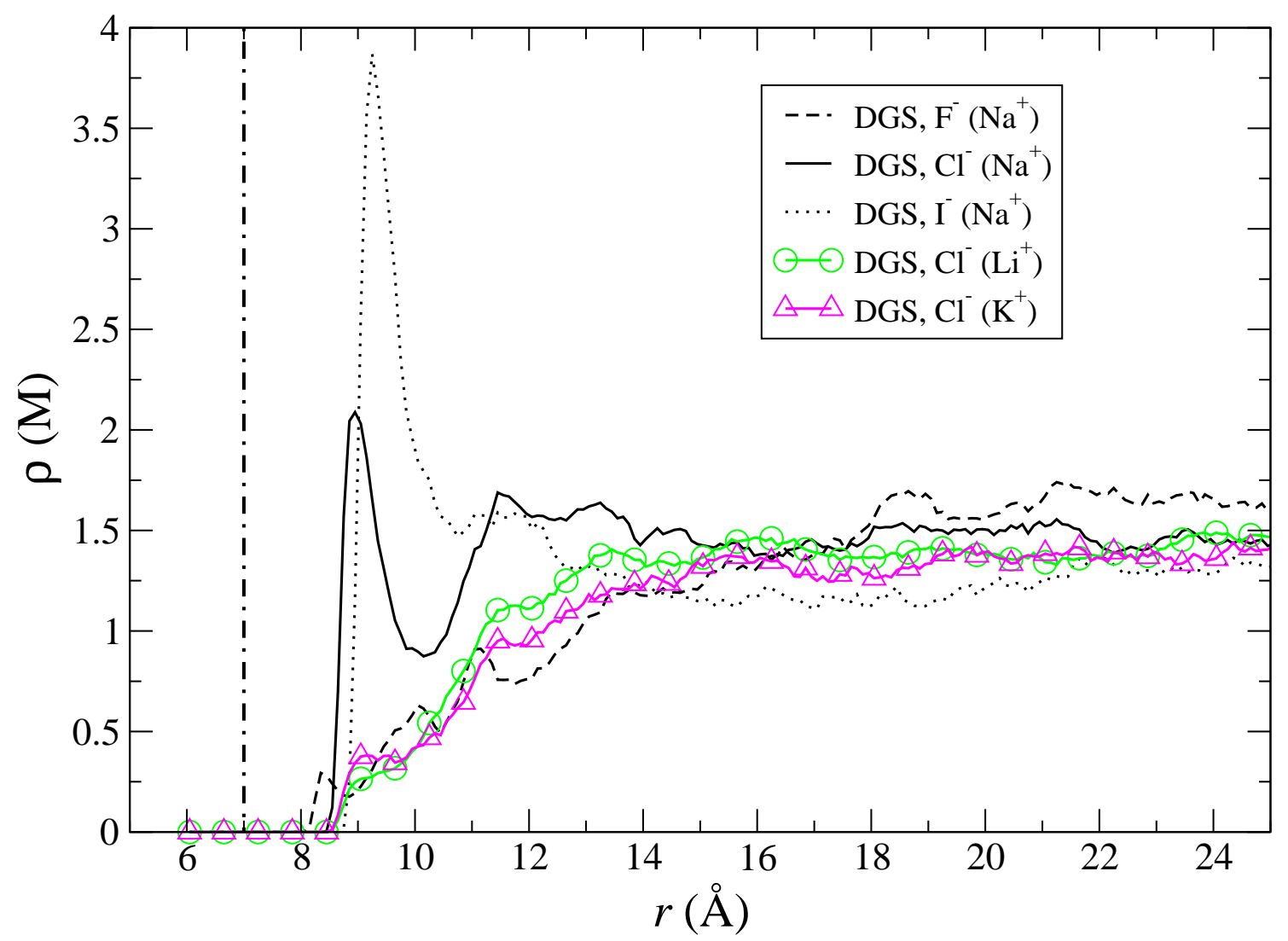

FIG. 14: Radial density profiles of anions around a cylindrical solute of $\varepsilon_{i n}=5$ for various salts. 